\title{
The Full Second-Order Radiation Transfer Function for Large-Scale CMB Anisotropies
}

\author{
Nicola Bartolo* \\ The Abdus Salam International Centre for Theoretical Physics, Strada Costiera 11, 34100 Trieste, Italy \\ Sabino Matarrese ${ }^{\dagger}$ \\ Dipartimento di Fisica "G. Galilei", Università di Padova, \\ and INFN, Sezione di Padova, via Marzolo 8, Padova I-35131, Italy \\ Antonio Riotto $\ddagger$ \\ CERN, Theory Division, CH-1211 Geneva 23, Switzerland
}

(Dated: December 22, 2005)

\begin{abstract}
We calculate the full second-order radiation transfer function for Cosmic Microwave Background anisotropies on large angular scales in a flat universe filled with matter and cosmological constant. It includes $(i)$ the second-order generalization of the Sachs-Wolfe effect, and of (ii) both the early and late Integrated Sachs-Wolfe effects, $($ iii) the contribution of the second-order tensor modes, and is valid for a generic set of initial conditions specifying the level of primordial non-Gaussianity.
\end{abstract}

PACS numbers: $98.80 . \mathrm{Cq}$

DFPD-A/05/23

\section{INTRODUCTION}

Cosmological inflation [1] has become the dominant paradigm to understand the initial conditions for Cosmic Microwave Background ( $\mathrm{CMB}$ ) anisotropies and structure formation. In the inflationary picture, the primordial cosmological perturbations are created from quantum fluctuations "redshifted" out of the horizon during an early period of accelerated expansion of the universe, where they remain "frozen". They are observable as temperature anisotropies in the CMB. They were first detected by the COsmic Background Explorer (COBE) satellite [2, 3, 4]and then with spectacular accuracy most recently by the the Wilkinson Microwave Anisotropy Probe (WMAP) mission $[5,6]$. Since the observed cosmological perturbations are of the order of $10^{-5}$, one might think that first-order perturbation theory will be adequate for all comparison with observations. That may not be the case however, as the Planck satellite [7] and its successors may be sensitive to the non-Gaussianity of the cosmological perturbations at the level of second- or higher-order perturbation theory [8]. Statistics like the bispectrum and the trispectrum of the CMB can then be used to assess the level of primordial non-Gaussianity on various cosmological scales and to discriminate it from the one induced by secondary anisotropies and systematic effects $[8,9,10,11]$. Therefore, it is of fundamental importance to provide accurate theoretical predictions for the statistics of CMB anisotropies as left imprinted by the primordial seeds originated during or immediately after inflation. Steps towards this goal have been taken in Refs. $[12,13,14,15,16,17]$ at the level of second order perturbation theory.

In this paper we provide the first computation of the complete second-order radiation transfer function for CMB anisotropies on large angular scales in a flat $\Lambda$ Cold Dark Matter $(\Lambda \mathrm{CDM})$ model. Our calculation includes the second-order generalization of the Sachs-Wolfe as well as of the (both early and late) Integrated Sachs-Wolfe effects and is valid for a generic set of initial conditions specifying the level of primordial non-Gaussianity. It also accounts for the contribution of second-order tensor perturbations. Our results which are complete as fas as large angular scales are concerned, represent the first step towards the computation of the full second-order radiation transfer function for CMB anisotropies. Its computation also on small angular scales, which involves several non-linear effects, such as gravitational lensing, Shapiro time-delay, Rees-Sciama effects, etc. and, of course, dealing with the non-linear Boltzmann equation for the photon-matter fluid (some specific aspects of which have been studied in Ref. [18]), will be the subject of a future publication [19].

The general expression for CMB anisotropies up to second order from gravitational perturbations has been obtained in Refs. [12, 13]. At large angular scales the extension up to second order of the Sachs-Wolfe effect [20] has been derived in the Poisson gauge (for adiabatic perturbations) in Refs. [8, 21] (and for a gauge-invariant generalization at

\footnotetext{
*Electronic address: nbartolo@ictp.trieste.it

†Electronic address: sabino.matarrese@pd.infn.it

${ }^{\ddagger}$ Electronic address: antonio.riotto@pd.infn.it
} 
second-order see Ref. [14]) ${ }^{1}$

$$
\frac{\Delta T}{T}=\frac{1}{3} \phi_{*}-\frac{5}{18} \phi_{1 *}^{2},
$$

where $\phi_{*}=\phi_{1 *}+\phi_{2 *} / 2 \equiv \varphi_{*}+\phi_{2 *} / 2$ is the gravitational potential at emission on the last scattering surface and is split into linear and second-order parts (see Eq. (2.1)). Such an expression allows to single out the initial (at last scattering) non-linearities of the large-scale CMB anisotropies, including the primordial non-Gaussianity of cosmological perturbations, as it has been computed in Refs. [8, 14, 21, 22]. In Eq. (1.1) the large-scale second-order gravitational potential $\phi_{2 *}$ at emission contains the non-linearity arising from inflation and from the post-inflationary epoch yielding $[8,14,21,22]$

$$
\frac{\Delta T}{T}=\frac{1}{3} \varphi_{*}+\frac{1}{18} \varphi_{*}^{2}-\frac{\mathcal{K}}{10}-\frac{5}{9}\left(a_{\mathrm{nl}}-1\right) \varphi_{*}^{2},
$$

where

$$
\mathcal{K} \equiv 10 \nabla^{-4} \partial_{i} \partial^{j}\left(\partial^{i} \varphi_{*} \partial_{j} \varphi_{*}\right)-\nabla^{-2}\left(\frac{10}{3} \partial^{i} \varphi_{*} \partial_{i} \varphi_{*}\right),
$$

and $a_{\mathrm{nl}}$ is a parameter specifying the level of primordial non-Gaussianity which depends upon the particular scenario for the generation of cosmological perturbations, as we will discuss later in more detail. For example, for standard single-field slow-roll models of inflation $a_{\mathrm{nl}}=1+\mathcal{O}(\epsilon, \eta)[23,24,25]$, where $\epsilon$ and $\eta$ are the standard slow-roll parameters (see e.g. Ref. [1]).

In this work we will focus on another contribution to the large-scale CMB anisotropies which is due to the evolution of the gravitational potentials from the epoch of last scattering up to now and it is encoded in a number of integrated terms [13]

$$
\frac{\Delta T}{T}(\hat{\mathbf{n}})=\left.\int_{\eta_{*}}^{\eta_{0}} d \eta \frac{\partial}{\partial \eta}(\phi+\psi)(\mathbf{x}, \eta)\right|_{\mathbf{x}=-\hat{\mathbf{n}}\left(\eta_{0}-\eta\right)}+(\text { first }- \text { order })^{2}
$$

where $\phi=\phi_{1}+\phi_{2} / 2$ and $\psi=\psi_{1}+\psi_{2} / 2$. In Eq. (1.4) $\hat{\mathbf{n}}$ denotes the direction of photon momentum and $\eta$ is the conformal time, $\eta_{0}$ and $\eta_{*}$ being the present and the last scattering times, respectively. Note that the partial time derivative of the perturbations must be evaluated along the line of sight. These integrated terms represent the gravitational redshift due to the time variation of the metric during the photon travel from the last scattering surface to the observer. The linear contribution is the so-called Integrated Sachs-Wolfe (ISW) effect and in a flat matter dominated (Einstein-de Sitter) Universe it vanishes due to the constancy in time of the gravitational potentials. However, in the presence of a cosmological constant (or more general dark energy component) it actually gives a large-scale contribution due to the decay in time of the potentials: this is the so-called late ISW effect. The early ISW effect similarly arises because of the residual dynamical role of the radiation component at times close to the lastscattering epoch; this also makes the gravitational potentials evolve in time, leading to a non-negligible contribution to the large-scale anisotropy (see e.g. Ref. [26]). The second-order generalization of the ISW effect is characterized by some peculiar properties. Even in a flat matter-dominated Universe, when the linear counterpart vanishes because the gravitational potential is constant, the evolution of the perturbations in the non-linear regime generates a nonvanishing contribution to the CMB anisotropies, the so-called Rees-Sciama effect [27]. In this paper we will compute the second-order ISW generated in a universe with a non-vanishing cosmological constant term. Unlike the usual Rees-Sciama effect, a contribution to the CMB anisotropies on large scales is generated, and therefore for the rest of the paper by second-order ISW we will refer to the large scale part of Eq. (1.4). Such an effect has not been considered so far, and thus a major part of this paper will deal with its study. An important feature is that it is related to possible primordial non-Gaussianity in the energy-density perturbations produced in the early universe. In a separate paper we will give a quantitative study of its signatures in the bispectrum of the CMB anisotropies [19]. Notice that in Eq. (1.4) we have also indicated additional contributions to CMB anisotropies which arise from integrated terms quadratic in the linear metric perturbations $[12,13]$. We will discuss and take them into account later. Finally in writing Eq. (1.4) we have dropped possible gravitational redshifts from second-order vector modes which are expected to give a negligible contribution on large scales [13].

This paper serves for different purposes. First it contains an independent part about the evolution of the secondoder perturbations in a flat universe filled with matter and a cosmological constant term. Second it discusses the

\footnotetext{
${ }^{1}$ For a fully non-linear generalization of the Sachs-Wolfe effect see Ref. [22].
} 
generation of CMB anisotropies due to the second-order ISW and its connection to primordial non-Gaussianity. The final achievement is that of providing the full second-order radiation transfer function for CMB anisotropies on large angular scales from the second-order Sachs-Wolfe and ISW effects. Our formalism is the starting point of an accurate evaluation of different effects on the CMB anisotropies, including the exact expression for the CMB bispectrum on large scales [19].

The paper is organized as follows. In Section II we present the main equations and solutions for the evolution of the gravitational potentials in a $\Lambda$ CDM universe, accounting for primordial non-Gaussianity in the initial conditions (see also Appendix A). In Section III we derive the expression for the second-order ISW by taking the large-scale contributions to Eq. (1.4) and we derive an expression of the CMB anisotropies expanded in multipoles (with details in Appendix B). In Section IV we study the contribution to the CMB anisotropies from second-order tensor modes. Finally in Section V we provide the full expression of the second-order radiation transfer function for large-scale CMB anisotropies. In this section we summarize our final formulae as a list and we provide the reader with all the necessary definitions. Section V contains also our conclusions.

\section{SECOND-ORDER PERTURBATIONS OF A FLAT $\Lambda$ CDM UNIVERSE IN THE POISSON GAUGE}

\section{A. Evolution of the second-order gravitational potentials}

We consider a spatially flat Universe filled with a cosmological constant $\Lambda$ and a non-relativistic pressureless fluid of Cold Dark Matter (CDM) plus baryons, whose energy-momentum tensor reads $T_{\nu}^{\mu}=\rho u^{\mu} u_{\nu}$. The radiation component, which also plays a role during the early stages following the last-scattering epoch, will be considered in Section IIIC, in connection with the early ISW effect. We will work in the so-called Poisson gauge which, to linear order, reduces to the Newtonian gauge. Using the conformal time $d \eta=d t / a$, the perturbed line element around a spatially flat FRW background reads

$$
d s^{2}=a^{2}(\eta)\left\{-(1+2 \phi) d \eta^{2}+2 \omega_{i} d \eta d x^{i}+\left[(1-2 \psi) \delta_{i j}+\chi_{i j}\right] d x^{i} d x^{j}\right\} .
$$

Here each perturbation quantity is expanded into a first-order (linear) part and a second-order contribution in the same way as for the gravitational potential $\phi=\phi_{1}+\phi_{2} / 2$. In the Poisson gauge the shift perturbation $\omega_{i}$ is a pure vector, i.e. $\partial_{i} \omega^{i}=0$, while $\chi_{i j}$ is a tensor mode (i.e. divergence-free and traceless $\partial^{i} \chi_{i j}=0, \chi_{i}^{i}=0$ ). In this paper we are mainly interested in the evolution of the second-order scalar potentials. Moreover we will neglect linear vector modes since they are not produced in standard mechanisms for the generation of cosmological perturbations (as inflation), and we also neglect tensor modes at linear order, since they give a negligible contribution to the bispectrum arising from the integrated effects (1.4). In Section IV and Appendix A, we will report also the computation for the second-order vector and tensor modes following the results of Ref. [28]. As for the matter component we split the mass density into a homogeneous $\bar{\rho}(\eta)$ and a perturbed part as $\rho(\mathbf{x}, \eta)=\bar{\rho}(\eta)\left(1+\delta_{1}+\delta_{2} / 2\right)$ and we write the four velocity as $u^{\mu}=\left(\delta_{0}^{\mu}+v^{\mu}\right) / a$ with $u^{\mu} u_{\mu}=-1$ and $v^{\mu}=v_{1}^{\mu}+v_{2}^{\mu} / 2$.

The Friedmann background equations are $3 \mathcal{H}^{2}=a^{2}(8 \pi G \bar{\rho}+\Lambda)$ and $\bar{\rho}^{\prime}=-3 \mathcal{H} \bar{\rho}$, where a prime stands for differentiation with respect to conformal time, and $\mathcal{H}=a^{\prime} / a$.

Let us briefly recall the results for the linear perturbations in the case of a non-vanishing cosmological $\Lambda$ term. At linear order the traceless part of the $(i-j)$-components of Einstein equations gives $\phi_{1}=\psi_{1} \equiv \varphi$. Its trace gives the evolution equation for the linear scalar potential $\varphi$

$$
\varphi^{\prime \prime}+3 \mathcal{H} \varphi^{\prime}+a^{2} \Lambda \varphi=0 .
$$

Selecting only the growing mode solution one can write

$$
\varphi(\mathbf{x}, \eta)=g(\eta) \varphi_{0}(\mathbf{x})
$$

where $\varphi_{0}$ is the peculiar gravitational potential linearly extrapolated to the present time $\left(\eta_{0}\right)$ and $g(\eta)=D_{+}(\eta) / a(\eta)$ is the so called growth-suppression factor, where $D_{+}(\eta)$ is the linear growing-mode of density fluctuations in the Newtonian limit. The exact form of $g$ can be found in Refs. [29, 30,31]. In the $\Lambda=0$ case $g=1$. A very good approximation for $g$ as a function of redshift $z$ is given in Refs. [29, 30]

$$
g \propto \Omega_{m}\left[\Omega_{m}^{4 / 7}-\Omega_{\Lambda}+\left(1+\Omega_{m} / 2\right)\left(1+\Omega_{\Lambda} / 70\right)\right]^{-1}
$$

with $\Omega_{m}=\Omega_{0 m}(1+z)^{3} / E^{2}(z), \Omega_{\Lambda}=\Omega_{0 \Lambda} / E^{2}(z), E(z) \equiv(1+z) \mathcal{H}(z) / \mathcal{H}_{0}=\left[\Omega_{0 m}(1+z)^{3}+\Omega_{0 \Lambda}\right]^{1 / 2}$ and $\Omega_{0 m}$, $\Omega_{0 \Lambda}=1-\Omega_{0 m}$, the present-day density parameters of non-relativistic matter and cosmological constant, respectively. We will normalize the growth-suppression factor so that $g(z=0)=1$. 
Let us now consider the second-order scalar perturbations. For simplicity, we adopt the notation

$$
\begin{aligned}
\phi_{2} & \equiv \Phi, \\
\psi_{2} & \equiv \Psi
\end{aligned}
$$

The evolution equation for the second-order gravitational potential $\Psi$ is obtained from the trace of the $(i-j)$-Einstein equations: its detailed derivation will be given in Appendix A; here we just report it

$$
\Psi^{\prime \prime}+3 \mathcal{H} \Psi^{\prime}+a^{2} \Lambda \Psi=S(\eta)
$$

where $S(\eta)$ is the source term

$$
\begin{aligned}
S(\eta) & =g^{2} \Omega_{m} \mathcal{H}^{2}\left[\frac{(f-1)^{2}}{\Omega_{m}} \varphi_{0}^{2}+2\left(2 \frac{(f-1)^{2}}{\Omega_{m}}-\frac{3}{\Omega_{m}}+3\right) \times\left(\nabla^{-2}\left(\partial^{i} \varphi_{0} \partial_{i} \varphi_{0}\right)-3 \nabla^{-4} \partial_{i} \partial^{j}\left(\partial^{i} \varphi_{0} \partial_{j} \varphi_{0}\right)\right)\right] \\
& +g^{2}\left[\frac{4}{3}\left(\frac{f^{2}}{\Omega_{m}}+\frac{3}{2}\right) \nabla^{-2} \partial_{i} \partial^{j}\left(\partial^{i} \varphi_{0} \partial_{j} \varphi_{0}\right)-\left(\partial^{i} \varphi_{0} \partial_{i} \varphi_{0}\right)\right],
\end{aligned}
$$

and we have introduced the function

$$
f(\eta)=\frac{d \ln D_{+}}{d \ln a}=1+\frac{g^{\prime}(\eta)}{\mathcal{H} g(\eta)}
$$

which can be written as a function of $\Omega_{m}$ as $f\left(\Omega_{m}\right) \approx \Omega_{m}(z)^{4 / 7}[29,30]$. In Eq. (2.8) $\nabla^{-2}$ stands for the inverse of the Laplacian operator.

The solution of Eq. (2.7) is then given by

$$
\Psi(\eta)=\frac{g}{g_{m}} \Psi_{m}+\Psi_{+}(\eta) \int_{\eta_{m}}^{\eta} d \eta^{\prime} \frac{\Psi_{-}\left(\eta^{\prime}\right)}{W\left(\eta^{\prime}\right)} S\left(\eta^{\prime}\right)-\Psi_{-}(\eta) \int_{\eta_{m}}^{\eta} d \eta^{\prime} \frac{\Psi_{+}\left(\eta^{\prime}\right)}{W\left(\eta^{\prime}\right)} S\left(\eta^{\prime}\right) .
$$

Here $\Psi_{+}(\eta)=g(\eta)$ and $\Psi_{-}(\eta)=\mathcal{H}(\eta) / a^{2}(\eta)$ are the solutions of the homogeneous equation, $W$ is the Wronskian which explicitly reads $W(\eta)=W_{0} / a^{3}$ with $W_{0}=\mathcal{H}_{0}^{2}\left(f_{0}+(3 / 2) \Omega_{0 m}\right)$, where the suffix ' 0 ' stands for the value of the corresponding quantities at the present time. In particular notice that $\Psi_{m} \equiv \Psi\left(\eta_{m}\right)$ represents the initial condition taken deep in the matter dominated era on super-horizon scales, $\eta_{m}$ being the epoch when full matter domination starts. It is such an initial value that must be properly determined in order to account for the primordial nonGaussianity in the cosmological perturbations. The evolution of the gravitational potential $\Phi$ is then obtained from the relation between $\Psi$ and $\Phi$

$$
\nabla^{2} \nabla^{2} \Psi=\nabla^{2} \nabla^{2} \Phi-4 g^{2} \nabla^{2} \nabla^{2} \varphi_{0}^{2}-\frac{4}{3} g^{2}\left(\frac{f^{2}}{\Omega_{m}}+\frac{3}{2}\right)\left[\nabla^{2}\left(\partial_{i} \varphi_{0} \partial^{i} \varphi_{0}\right)-3 \partial_{i} \partial^{j}\left(\partial^{i} \varphi_{0} \partial_{j} \varphi_{0}\right)\right]
$$

which follows from the traceless part of the $(i-j)$-component of Einstein equations (see Appendix A). In particular deep in the matter dominated epoch Eq. (2.11) reduces to

$$
\nabla^{2} \nabla^{2} \Psi_{m}=\nabla^{2} \nabla^{2} \Phi_{m}-4 g_{m}^{2} \nabla^{2} \nabla^{2} \varphi_{0}^{2}-\frac{10}{3} g_{m}^{2}\left[\nabla^{2}\left(\partial^{i} \varphi_{0} \partial_{i} \varphi_{0}\right)-3 \partial^{i} \partial_{j}\left(\partial_{i} \varphi_{0} \partial^{j} \varphi_{0}\right)\right]
$$

where we have used that for $\Omega_{m} \rightarrow 1 f(\eta) \rightarrow 1$, and where $\varphi_{m}=g_{m} \varphi_{0}$ is the value of the gravitational potential during matter domination, when the cosmological constant was still negligible, with $g_{m}=g\left(\eta_{m}\right){ }^{2}$ Note that in this way Eq. (2.12) reduces to the relation for the two gravitational potentials $\Phi$ and $\Psi$ first obtained in Ref. [25] for a matter-dominated Universe.

\section{B. Initial conditions from primordial non-Gaussianity}

Let us now discuss the key issue of the initial conditions which we conveniently fix at the time when the cosmological perturbations relevant today for Large Scale Structures and CMB anisotropies are well outside the Hubble radius, $i$.e.

\footnotetext{
${ }^{2}$ A good approximation, $g_{m} \approx \frac{2}{5} \Omega_{0 m}^{-1}\left(\Omega_{0 m}^{4 / 7}+\frac{3}{2} \Omega_{0 m}\right)$.
} 
when the (comoving) wavelength $\lambda$ of a given perturbation mode is such that $\lambda \gg \mathcal{H}^{-1}, \mathcal{H}=a^{\prime} / a$ being the horizon size.

In the standard single-field inflationary model, the first seeds of density fluctuations are generated on super-horizon scales from the fluctuations of a scalar field, the inflaton [1]. Recently many other scenarios have been proposed as alternative mechanisms to generate such primordial seeds. These include, for example, the curvaton [32] and the inhomogeneous reheating scenarios [33], where essentially the first density fluctuations are produced through the fluctuations of a scalar field other than the inflaton. In order to follow the evolution on super-horizon scales of the density fluctuations coming from the various mechanisms, we use the curvature perturbation of uniform density hypersurfaces $\zeta=\zeta_{1}+\zeta_{2} / 2+\cdots$, where $\zeta_{1}=-\psi_{1}-\mathcal{H} \delta \rho_{1} / \bar{\rho}^{\prime}$ and the expression for $\zeta_{2}$ is given by [34]

$$
\zeta_{2}=-\psi_{2}-\mathcal{H} \frac{\delta_{2} \rho}{\rho^{\prime}}+\Delta \zeta_{2}
$$

with

$$
\Delta \zeta_{2}=2 \mathcal{H} \frac{\delta_{1} \rho^{\prime}}{\rho^{\prime}} \frac{\delta_{1} \rho}{\rho^{\prime}}+2 \frac{\delta_{1} \rho}{\rho^{\prime}}\left(\psi_{1}^{\prime}+2 \mathcal{H} \psi_{1}\right)-\left(\frac{\delta_{1} \rho}{\rho^{\prime}}\right)^{2}\left(\mathcal{H} \frac{\rho^{\prime \prime}}{\rho}-\mathcal{H}^{\prime}-2 \mathcal{H}^{2}\right)
$$

The crucial point is that the gauge-invariant curvature perturbation $\zeta$ remains constant on super-horizon scales after it has been generated during a primordial epoch and possible isocurvature perturbations are no longer present. Therefore, we may set the initial conditions at the time when $\zeta$ becomes constant. In particular, $\zeta_{2}$ provides the necessary information about the "primordial" level of non-Gaussianity generated either during inflation, as in the standard scenario, or immediately after it, as in the curvaton scenario. Different scenarios are characterized by different values of $\zeta_{2}$. For example, in the standard single-field inflationary model $\zeta_{2}=2\left(\zeta_{1}\right)^{2}+\mathcal{O}(\epsilon, \eta)[23,25]$, where $\epsilon$ and $\eta$ are the standard slow-roll parameters [1]. In general, we may parametrize the primordial non-Gaussianity level in terms of the conserved curvature perturbation as in Ref. [14]

$$
\zeta_{2}=2 a_{\mathrm{nl}}\left(\zeta_{1}\right)^{2}
$$

where the parameter $a_{\mathrm{nl}}$ depends on the physics of a given scenario. For example in the standard scenario $a_{\mathrm{nl}} \simeq 1$, while in the curvaton case $a_{\mathrm{nl}}=(3 / 4 r)-r / 2$, where $r \approx\left(\rho_{\sigma} / \rho\right)_{\mathrm{D}}$ is the relative curvaton contribution to the total energy density at curvaton decay $[8,35]$. In the minimal picture for the inhomogeneous reheating scenario, $a_{\mathrm{nl}}=1 / 4$. For other scenarios we refer the reader to Ref. [8]. One of the best tools to detect or constrain the primordial large-scale non-Gaussianity is through the analysis of the CMB anisotropies, for example by studying the bispectrum [8]. In that case the standard procedure is to introduce the non-linearity parameter $f_{\mathrm{nl}}$ characterizing non-Gaussianity in the large-scale temperature anisotropies $[8,36,37]$. To give the feeling of the resulting size of $f_{\mathrm{nl}}$ when $\left|a_{\mathrm{nl}}\right| \gg 1, f_{\mathrm{nl}} \simeq 5 a_{\mathrm{nl}} / 3$ (see Refs. [8, 14]). The conserved value of the curvature perturbation $\zeta$ allows to set the initial conditions for the metric and matter perturbations accounting for the primordial contributions. At linear order during the matter-dominated epoch and on large scales $\zeta_{1}=-5 \varphi_{m} / 3$, where $\varphi_{m}=g_{m} \varphi_{0}$ is the value of the gravitational potential during matter domination, when the cosmological constant was still negligible. Thus we can write

$$
\zeta_{2}=\frac{50}{9} a_{\mathrm{nl}} \varphi_{m}^{2}=\frac{50}{9} a_{\mathrm{nl}} g_{m}^{2} \varphi_{0}^{2}
$$

On the other hand by using the expression for $\zeta_{2}$ during the matter-dominated epoch together with Eq. (2.12) and the second-order (0-0)-component of Einstein equations (evaluated for a matter-dominated epoch), one can express $\Phi_{m}$ in terms of $\zeta_{2}$ as (see Refs. [8, 14, 21, 25])

$$
\Phi_{m}=-\frac{3}{5} \zeta_{2}+\frac{16}{3} \varphi_{m}^{2}+2 \nabla^{-2}\left(\partial^{i} \varphi_{m} \partial_{i} \varphi_{m}\right)-6 \nabla^{-4} \partial_{i} \partial^{j}\left(\partial^{i} \varphi_{m} \partial_{j} \varphi_{m}\right)
$$

Therefore using Eq. (2.16) we find, for generic non-Gaussian initial conditions parametrized by $\zeta_{2}=2 a_{\mathrm{nl}}\left(\zeta_{1}\right)^{2}$,

$$
\begin{aligned}
& \Phi_{m}=2 g_{m}^{2}\left[\left(-\frac{5}{3}\left(a_{\mathrm{nl}}-1\right)+1\right) \varphi_{0}^{2}+\nabla^{-2}\left(\partial^{i} \varphi_{0} \partial_{i} \varphi_{0}\right)-3 \nabla^{-4} \partial_{i} \partial^{j}\left(\partial^{i} \varphi_{0} \partial_{j} \varphi_{0}\right)\right], \\
& \Psi_{m}=2 g_{m}^{2}\left[\left(-\frac{5}{3}\left(a_{\mathrm{nl}}-1\right)-1\right) \varphi_{0}^{2}-\frac{2}{3}\left(\nabla^{-2}\left(\partial^{i} \varphi_{0} \partial_{i} \varphi_{0}\right)-3 \nabla^{-4} \partial_{i} \partial^{j}\left(\partial^{i} \varphi_{0} \partial_{j} \varphi_{0}\right)\right)\right],
\end{aligned}
$$


where $a_{\mathrm{nl}}$ will always signal the presence of primordial non-Gaussianity according to our parametrization. The last of these equations (2.19) has been obtained from Eq. (2.12).

After having determined the initial conditions from Eq. (2.10) we finally get

$$
\begin{aligned}
\Psi(\eta) & =2 g(\eta) g_{m}\left[\left(-\frac{5}{3}\left(a_{\mathrm{nl}}-1\right)-1\right) \varphi_{0}^{2}-\frac{2}{3}\left(\nabla^{-2}\left(\partial^{i} \varphi_{0} \partial_{i} \varphi_{0}\right)-3 \nabla^{-4} \partial_{i} \partial^{j}\left(\partial^{i} \varphi_{0} \partial_{j} \varphi_{0}\right)\right)\right] \\
& +\mathcal{H}_{0}^{-2}\left(f_{0}+\frac{3}{2} \Omega_{0 m}\right)^{-1}\left[g(\eta) \int_{\eta_{m}}^{\eta} d \tilde{\eta} a(\tilde{\eta}) \mathcal{H}(\tilde{\eta}) S(\tilde{\eta})-\frac{\mathcal{H}(\eta)}{a^{2}(\eta)} \int_{\eta_{m}}^{\eta} d \tilde{\eta} a^{3}(\tilde{\eta}) g(\tilde{\eta}) S(\tilde{\eta})\right], \\
\Phi(\eta) & =\Psi(\eta)+4 g^{2}(\eta) \varphi_{0}^{2}+\frac{4}{3} g^{2}(\eta)\left(\frac{f^{2}}{\Omega_{m}}+\frac{3}{2}\right)\left[\nabla^{-2}\left(\partial^{i} \varphi_{0} \partial_{i} \varphi_{0}\right)-3 \nabla^{-4} \partial_{i} \partial^{j}\left(\partial^{i} \varphi_{0} \partial_{j} \varphi_{0}\right)\right] .
\end{aligned}
$$

Notice that we can rewrite the solutions for $\Psi$ and $\Phi$ in a compact form as

$$
\begin{aligned}
\Psi(\eta) & =\left(B_{1}(\eta)-2 g(\eta) g_{m}-\frac{10}{3}\left(a_{\mathrm{nl}}-1\right) g(\eta) g_{m}\right) \varphi_{0}^{2}+\left(B_{2}(\eta)-\frac{4}{3} g(\eta) g_{m}\right)\left[\nabla^{-2}\left(\partial^{i} \varphi_{0} \partial_{i} \varphi_{0}\right)-3 \nabla^{-4} \partial_{i} \partial^{j}\left(\partial^{i} \varphi_{0} \partial_{j} \varphi_{0}\right)\right] \\
& +B_{3}(\eta) \nabla^{-2} \partial_{i} \partial^{j}\left(\partial^{i} \varphi_{0} \partial_{j} \varphi_{0}\right)+B_{4}(\eta) \partial^{i} \varphi_{0} \partial_{i} \varphi_{0} \\
\Phi(\eta) & =\left(B_{1}(\eta)+4 g^{2}(\eta)-2 g(\eta) g_{m}-\frac{10}{3}\left(a_{\mathrm{nl}}-1\right) g(\eta) g_{m}\right) \varphi_{0}^{2}+\left[B_{2}(\eta)+\frac{4}{3} g^{2}(\eta)\left(e(\eta)+\frac{3}{2}\right)-\frac{4}{3} g(\eta) g_{m}\right] \\
& \times\left[\nabla^{-2}\left(\partial^{i} \varphi_{0} \partial_{i} \varphi_{0}\right)-3 \nabla^{-4} \partial_{i} \partial^{j}\left(\partial^{i} \varphi_{0} \partial_{j} \varphi_{0}\right)\right]+B_{3}(\eta) \nabla^{-2} \partial_{i} \partial^{j}\left(\partial^{i} \varphi_{0} \partial_{j} \varphi_{0}\right)+B_{4}(\eta) \partial^{i} \varphi_{0} \partial_{i} \varphi_{0},
\end{aligned}
$$

where we have introduced $B_{i}(\eta)=\mathcal{H}_{0}^{-2}\left(f_{0}+3 \Omega_{0 m} / 2\right)^{-1} \tilde{B}_{i}(\eta)$ with the following definitions

$$
\begin{array}{ll}
\tilde{B}_{1}(\eta)=\int_{\eta_{m}}^{\eta} d \tilde{\eta} \mathcal{H}^{2}(\tilde{\eta})(f(\tilde{\eta})-1)^{2} C(\eta, \tilde{\eta}), & \tilde{B}_{2}(\eta)=2 \int_{\eta_{m}}^{\eta} d \tilde{\eta} \mathcal{H}^{2}(\tilde{\eta})\left[2(f(\tilde{\eta})-1)^{2}-3+3 \Omega_{m}(\tilde{\eta})\right] C(\eta, \tilde{\eta}),(2 . \\
\tilde{B}_{3}(\eta)=\frac{4}{3} \int_{\eta_{m}}^{\eta} d \tilde{\eta}\left(e(\tilde{\eta})+\frac{3}{2}\right) C(\eta, \tilde{\eta}), & \tilde{B}_{4}(\eta)=-\int_{\eta_{m}}^{\eta} d \tilde{\eta} C(\eta, \tilde{\eta})
\end{array}
$$

and

$$
C(\eta, \tilde{\eta})=g^{2}(\tilde{\eta}) a(\tilde{\eta})\left[g(\eta) \mathcal{H}(\tilde{\eta})-g(\tilde{\eta}) \frac{a^{2}(\tilde{\eta})}{a^{2}(\eta)} \mathcal{H}(\eta)\right]
$$

with $e(\eta)=f^{2}(\eta) / \Omega_{m}(\eta)$.

Let us comment on the results found here, in order to proceed further by computing the radiation transfer function for the second-order $\mathrm{CMB}$ anisotropies on large scales. In the expression for the second-order gravitational potentials (2.22) and (2.23) we recognize two contributions. The term which dominates on small scales, $\left[B_{3}(\eta) \nabla^{-2} \partial_{i} \partial^{j}\left(\partial^{i} \varphi_{0} \partial_{j} \varphi_{0}\right)+B_{4}(\eta) \partial^{i} \varphi_{0} \partial_{i} \varphi_{0}\right]$, which gives rise to the second-order Newtonian piece and is insensitive to any non-linearity in the initial conditions. In fact, for a vanishing cosmological constant $\Lambda \rightarrow 0$, it is just this term which is responsible for the Rees-Sciama effect due to the non-linear evolution of the gravitational potentials. For the Einstein-de Sitter case, the signatures of the Newtonian Rees-Sciama effect in terms of the CMB bispectrum have been widely discussed in the literature $[38,39]$. An analysis of the CMB bispectrum induced by the Newtonian Rees-Sciama effect in the presence of a non-vanishing cosmological constant $\Lambda \neq 0$ has been given in Refs. [40,41].

The remaining pieces in Eqs. (2.22) and (2.23) represent the novelty of our work. They correspond to contributions which tend to dominate on large scales with respect to those characterizing the Newtonian contribution, and whose origin is purely relativistic. The contribution of these terms to the CMB anisotropies through Eq. (1.4) will be mainly on large scales through the time dependence of the growth-suppression factor $g(\eta)$ and it corresponds to the late ISW effect at second order. Moreover these are the pieces carrying the information on primordial non-Gaussianity, since they properly take into account in the initial conditions the primordial non-Gaussianity generated during inflation, as in the standard scenario, or immediately after it, as in the curvaton scenario. Terms of this type have been obtained for a flat matter-dominated (Einstein-de Sitter) Universe in Refs. [13, 42], but in this case there is no large-scale contribution through the integrated effect (1.4), because the different terms are constant in time, since $g(\eta)=1$ and $B_{1}(\eta)=B_{2}(\eta)=0$. In Ref. [15] second-order cosmological perturbations have been computed in the $\Lambda \neq 0$ case from the synchronous to the Poisson gauge, thus extending the analysis of Ref. [42], and the CMB temperature 
anisotropies induced by metric perturbations have been also considered by applying the expressions of Ref. [13]. However, an important point to notice is that both Refs. [13, 42] and Ref. [15] disregard any primordial non-linear contribution from inflation. ${ }^{3}$

\section{SECOND-ORDER ISW EFFECT IN A $\Lambda$ CDM UNIVERSE: PRIMORDIAL NON-GAUSSIANITY AND SECOND-ORDER RADIATION TRANSFER FUNCTION}

\section{A. Temperature anisotropies}

In order to give an expression for the second-order Integrated Sachs-Wolfe effect we will take the large-scale contributions to Eq. (1.4). Thus in Eqs. (2.22),(2.23) for the second-order gravitational potentials we will drop the Newtonian terms that have already been examined in Refs. [40, 41] in connection to the Rees-Sciama effect. Accordingly we will take the second-order corrections (first - order) ${ }^{2}$ - quadratic in the linear perturbations - appearing in Eq. (1.4) from Refs. $[12,13]$ following the criteria that they must include integrated contributions which survive on large scales.

Let us discuss this step in some details. From Refs. [12, 13] we are left to take the large-scale limit of the following expression

$$
(\text { first }- \text { order })^{2} \equiv \int_{\eta_{*}}^{\eta_{0}} d \eta\left[4 k^{(1) 0} \varphi^{\prime}+4 \varphi^{\prime} \varphi+2 x^{(1) 0} \varphi^{\prime \prime}+2 x^{(1) i} \varphi_{, i}^{\prime}\right]-I_{1}\left(\eta_{*}\right)\left(\varphi_{*}+\tau_{1 *}-I_{1}\left(\eta_{*}\right)\right) .
$$

In Eq. (3.1) $\tau_{1 *}$ is the linear fractional intrinsic temperature fluctuation on the last scattering surface, and $I_{1}\left(\eta_{*}\right)$ is minus the linear ISW effect, with

$$
I_{1}(\eta)=2 \int_{\eta_{0}}^{\eta} d \tilde{\eta} \varphi^{\prime}
$$

while $k^{(1) 0}$ and $x^{(1) \mu}$ are the first-order perturbation of the photon wavevector and background geodesic respectively $[12,13]$

$$
\begin{aligned}
& k^{(1) 0}(\eta)=-2 \varphi+I_{1}(\eta), \\
& x^{(1) 0}(\eta)=2 \int_{\eta_{0}}^{\eta} d \tilde{\eta}\left[-\varphi+(\eta-\tilde{\eta}) \varphi^{\prime}\right], \\
& x^{(1) i}(\eta)=-2 \int_{\eta_{0}}^{\eta_{*}} d \eta\left[\varphi e^{i}+(\eta-\tilde{\eta}) \varphi^{, i}\right],
\end{aligned}
$$

where $e^{i}=-n^{i}$ is the unit vector specifying the line-of-sight direction and we have dropped monopole terms due to the observer. In Eqs. (3.1)-(3.5) the integrands must be evaluated along the background geodesic $\left(\eta,\left(\eta_{0}-\eta\right) e^{i}\right), e . g$. in Eq. (3.2) $\varphi^{\prime} \equiv \varphi^{\prime}\left(\tilde{\eta}, \mathbf{x}=-\hat{\mathbf{n}}\left(\eta_{0}-\tilde{\eta}\right)\right)$, while a $*$ denotes quantities evaluated at the last scattering surface, e.g. $\varphi_{*} \equiv \varphi\left(\eta_{*}, \mathbf{x}=-\hat{\mathbf{n}}\left(\eta_{0}-\eta_{*}\right)\right)$.

Next, following Ref. [15] we notice that Eq. (3.1) can be further simplified. We use the relation

$$
x^{(1) 0}+x^{(1) i} e_{i}=-2 \int_{\eta_{0}}^{\eta} d \tilde{\eta} \varphi
$$

and the integration by parts

$$
2 \int_{\eta_{0}}^{\eta *} d \eta \varphi^{\prime} I_{1}(\eta)=\frac{1}{2}\left[I_{1}\left(\eta_{*}\right)\right]^{2}
$$

Therefore Eq. (3.1) becomes

$$
(\text { first }- \text { order })^{2}=-4 \int_{\eta_{*}}^{\eta_{0}} d \eta\left[\varphi \varphi^{\prime}+\varphi^{\prime \prime} \int_{\eta_{0}}^{\eta} d \tilde{\eta} \varphi\right]+\frac{1}{2}\left[I_{1}\left(\eta_{*}\right)\right]^{2}-I_{1}\left(\eta_{*}\right)\left(\tau_{1 *}+\varphi_{*}\right)-4 \varphi_{*}^{\prime} \int_{\eta_{*}}^{\eta_{0}} d \eta\left(\eta_{*}-\eta\right) \varphi,(3 .
$$

\footnotetext{
3 The results in Refs. [13, 15, 42] have initial conditions corresponding to our $a_{\mathrm{nl}}=0$.
} 
where we used the decomposition of the total derivative along the background geodesic for a generic function $f\left(\eta, x^{i}(\eta)\right), f^{\prime}=\partial f / \partial \eta=d f / d \eta+\partial_{i} f e^{i}$. In the following we will use the large-scale solution $\tau_{1 *}=-2 \varphi_{*} / 3$.

We are now able to provide an expression for the second-order ISW effect from Eq. (1.4). It is separated into two parts, the early ISW effect due to a non-negligible radiation component at last-scattering, and the late ISW due to the expansion growth suprresion in a $\Lambda$ dominated epoch. Correspondingly we will split each integral into two parts as $\int_{\eta_{*}}^{\eta_{0}} d \eta=\int_{\eta_{*}}^{\eta_{m}} d \eta+\int_{\eta_{m}}^{\eta_{0}} d \eta$, where $\eta_{m}$ represents the epoch when full matter domination is reached. Before that time the early ISW effect is in action. Therefore we write the second-order ISW effect from Eq. (1.4) as

$$
\frac{\Delta T_{2}}{T}(\mathbf{n})={\frac{\Delta T_{2}}{T}}^{\text {early }}+{\frac{\Delta T_{2}}{T}}^{\text {late }},
$$

with

$$
\frac{1}{2}{\frac{\Delta T_{2}}{T}}^{\text {early }}=\left.\frac{1}{2} \int_{\eta_{*}}^{\eta_{m}} d \eta(\Phi+\Psi)^{\prime}(\mathbf{x}, \eta)\right|_{\mathbf{x}=-\hat{\mathbf{n}}\left(\eta_{0}-\eta\right)}+(\text { first }- \text { order })_{\text {early }}^{2},
$$

where the (first - order) $)_{\text {early }}^{2}$ corrections are obtained from Eq. (3.8) by keeping all those contributions which would vanish in the limit of full matter domination at the epoch of last scattering. We will discuss the early ISW effect in details in section III C.

In the following we will focus on the late ISW effect

$$
\frac{1}{2}{\frac{\Delta T_{2}}{T}}^{\text {late }}=\left.\frac{1}{2} \int_{\eta_{m}}^{\eta_{0}} d \eta(\Phi+\Psi)^{\prime}(\mathbf{x}, \eta)\right|_{\mathbf{x}=-\hat{\mathbf{n}}\left(\eta_{0}-\eta\right)}+(\text { first }- \text { order })_{\text {late }}^{2}
$$

using the results of Sec. II. Taking the time derivative of Eqs. (2.22) and (2.23) the late second-order Integrated Sachs-Wolfe effect is given by

$$
\begin{aligned}
\frac{1}{2} \frac{\Delta T_{2}^{\text {late }}}{T}=\left.\int_{\eta_{m}}^{\eta_{0}} d \eta\left[2\left(-1-\frac{5}{3}\left(a_{\mathrm{nl}}-1\right)\right) g_{m} g^{\prime}(\eta)+B_{1}^{\prime}(\eta)+4 g(\eta) g^{\prime}(\eta)\right] \varphi_{0}^{2}\right|_{\mathbf{x}=-\hat{\mathbf{n}}\left(\eta_{0}-\eta\right)} \\
+\left.\int_{\eta_{m}}^{\eta_{0}} d \eta g_{m}^{2} \bar{B}(\eta)\left[\nabla^{-2}\left(\partial^{i} \varphi_{0} \partial_{i} \varphi_{0}\right)-3 \nabla^{-4} \partial_{i} \partial^{j}\left(\partial^{i} \varphi_{0} \partial_{j} \varphi_{0}\right)\right]\right|_{\mathbf{x}=-\hat{\mathbf{n}}\left(\eta_{0}-\eta\right)}+(\text { first }- \text { order })_{\text {late }}^{2} \cdot(3 .
\end{aligned}
$$

We recall that in order to obtain the expression (3.12) we have dropped from Eqs. (2.22) and (2.23) the terms $\left[B_{3}(\eta) \nabla^{-2} \partial_{i} \partial^{j}\left(\partial^{i} \varphi_{0} \partial_{j} \varphi_{0}\right)+B_{4}(\eta) \partial^{i} \varphi_{0} \partial_{i} \varphi_{0}\right]$, which give rise to the second-order Newtonian piece and give a negligible contribution on large scales, and in Eq. (3.12) the (first - order) $)_{\text {late }}^{2}$ terms are obtained by taking that part of Eq. (3.8) which is due to late integrated effects

$$
(\text { first }- \text { order })_{\text {late }}^{2}=-4 \int_{\eta_{m}}^{\eta_{0}} d \eta\left[\varphi \varphi^{\prime}+\varphi^{\prime \prime} \int_{\eta_{0}}^{\eta} d \tilde{\eta} \varphi\right]+\frac{1}{2}\left[I_{1}\left(\eta_{m}\right)\right]^{2}-\frac{1}{3} \varphi_{*} I_{1}\left(\eta_{m}\right) .
$$

For simplicity of notation in Eq. (3.12) we have introduced

$$
\bar{B}(\eta)=\left(\frac{B_{2}^{\prime}(\eta)}{g_{m}^{2}}-\frac{4}{3} \frac{g^{\prime}(\eta)}{g_{m}}\right)+\frac{4}{3} \frac{g^{\prime}(\eta) g(\eta)}{g_{m}^{2}}\left(e(\eta)+\frac{3}{2}\right)+\frac{2}{3} \frac{g^{2}(\eta)}{g_{m}^{2}} e^{\prime}(\eta) .
$$

Eq. (3.12) is the starting point to evaluate the CMB bispectrum induced by the late Integrated Sachs-Wolfe effect which arises from the explicit time-dependence of the linear gravitational potentials during the late accelerated phase [19]. A simple but important comment about Eq. (3.12) is that the whole effect is vanishing in the case of a vanishing darkenergy component. Moreover we can identify two fundamental contributions. One, proportional to $\left[-5\left(a_{\mathrm{nl}}-1\right) / 3\right]$, is directly sensitive to the primordial non-Gaussianity set in the early Universe from inflation. The importance of this term comes from the observation that in the case of strong non-Gaussianity $\left(\left|a_{\mathrm{nl}}\right| \gg 1\right)$ the ISW on large scales can be strongly amplified. The remaining terms are due to the non-linear evolution of the gravitational potentials after inflation and to second order-corrections in the temperature anisotropies. In the case of a high level of primordial non-Gaussianity one would expect these terms not to give the dominant contribution. However accounting for them is crucial in the computation of the second-order radiation transfer function for CMB temperature anisotropies, as we will discuss later. 


\section{B. Angular decomposition}

In order to compute the angular CMB bispectrum from the second-order ISW effect let us start from the expression of the (today) observed CMB anisotropies expanded into spherical harmonics as it is standard in the literature, with multipoles $a_{l m}$ given by

$$
a_{\ell m}=\int d^{2} \hat{\mathbf{n}} \frac{\Delta T(\hat{\mathbf{n}})}{T} Y_{\ell m}^{*}(\hat{\mathbf{n}}) .
$$

In terms of the multipoles $a_{l m}$ the linear and non-linear parts of the temperature fluctuations correspond to a linear Gaussian part $a_{l m}^{\mathrm{L}}$ and a non-Gaussian contribution $a_{l m}^{\mathrm{NL}}$

$$
a_{\ell m}=a_{\ell m}^{\mathrm{L}}+a_{\ell m}^{\mathrm{NL}} .
$$

Before computing $a_{l m}^{\mathrm{NL}}$ for the second-order ISW effect let us recall briefly that at linear order the multipoles $a_{l m}^{\mathrm{L}}$ are expressed as (see, e.g [43])

$$
a_{\ell m}^{\mathrm{L}}=4 \pi(-i)^{l} \int \frac{d^{3} k}{(2 \pi)^{3}} \phi_{1 i}(\mathbf{k}) \Delta_{\ell}^{(1)}(k) Y_{\ell m}^{*}(\hat{\mathbf{k}})
$$

where $\Delta_{\ell}^{(1)}(k)$ is the linear radiation transfer function which describes the relations between the initial fluctuations $\phi_{1 i}(\mathbf{k})$ and the observed temperature anisotropies. In the following we take this initial value at the epoch of matter dominance $\phi_{1 i}(\mathbf{k})=\varphi_{m}(\mathbf{k})$. For the linear Sachs-Wolfe effect acting on large scales (small $k$ ) one finds $\Delta_{\ell}^{(1)}(k)=$ $j_{\ell}\left(k\left(\eta_{0}-\eta_{*}\right)\right) / 3$ where $j_{\ell}(x)$ are spherical Bessel functions of order $\ell$. For smaller scales one can compute $\Delta_{\ell}^{(1)}(k)$ with numerical codes such as CMBFAST [44]. Expressing the non-linear part $a_{\ell m}^{\mathrm{NL}}$ in terms of the initial potential fluctuations corresponds to obtain a second-order radiation transfer function. The computation of the radiation transfer function at second-order in perturbation theory is very useful in that it allows, for example, to see what are the effects of the non-linearities (set on super-horizon scales) at the recombination epoch on different scales of the CMB anisotropy pattern. In this paper we are able to compute the second-order radiation transfer function corresponding to the large angular scales of CMB anisotropies. For small scales (big $k$ ) the computation requires to solve the non-linear Boltzmann equations for the photon-matter fluid. A comment is in order here. Notice that the primordial non-Gaussianity contribution proportional to $\left[-5\left(a_{\mathrm{nl}}-1\right) / 3\right]$ will be transferred linearly to the CMB anisotropies, in that it is already a second-order contribution. Thus the crucial information to compute the secondorder transfer function is carried by the remaining contributions in Eq. (3.12), which so far have not been properly taken into account. ${ }^{4}$

Let us sketch how to obtain $a_{\ell m}^{\mathrm{NL}}$ for the late ISW effect (3.12) taking as an example just the term proportional to the initial non-Gaussianity $A(\hat{\mathbf{n}})=-\left.\int_{\eta_{m}}^{\eta_{0}} d \eta \frac{10}{3}\left(a_{\mathrm{nl}}-1\right) g_{m} g^{\prime}(\eta) \varphi_{0}^{2}\right|_{\mathbf{x}=-\hat{\mathbf{n}}\left(\eta_{0}-\eta\right)}$ in Eq. (3.12). Let us Fourier expand $A(\hat{\mathbf{n}})$

$$
A(\hat{\mathbf{n}})=\int \frac{d^{3} k}{(2 \pi)^{3}} \int_{\eta_{m}}^{\eta_{0}} d \eta \mathcal{A} g_{m} g^{\prime}(\eta)\left[\varphi_{0}^{2}\right](\mathbf{k}) e^{-i \mathbf{k} \cdot \hat{\mathbf{n}}\left(\eta_{0}-\eta\right)}
$$

where we set $\mathcal{A}=-10\left(a_{\mathrm{nl}}-1\right) / 3$ and $\left[\varphi_{0}^{2}\right](\mathbf{k})$ denotes the Fourier transform of $\varphi_{0}^{2}(\mathbf{x})$. If we now make use of the Legendre expansion $e^{i \mathbf{k} \cdot \mathbf{x}}=\sum_{\ell}(2 \ell+1) i^{\ell} j_{\ell}(k x) P_{\ell}(\hat{\mathbf{k}} \cdot \hat{\mathbf{x}})$ we find

$$
A(\hat{\mathbf{n}})=\int \frac{d^{3} k}{(2 \pi)^{3}} \sum_{\ell=0}^{\infty}(-i)^{\ell}(2 \ell+1) \int_{\eta_{m}}^{\eta_{0}} d \eta \mathcal{A} g_{m} g^{\prime}(\eta) j_{\ell}\left(k\left(\eta_{0}-\eta\right)\right)\left[\varphi_{0}^{2}\right](\mathbf{k}) P_{\ell}(\hat{\mathbf{k}} \cdot \hat{\mathbf{n}}) .
$$

Thus inserting Eq. (3.19) into Eq. (3.15) we get the corresponding $a_{\ell m}^{\mathrm{NL}}$

$$
a_{\ell m}^{\mathrm{NL}}(A)=4 \pi(-i)^{\ell} \int \frac{d^{3} k}{(2 \pi)^{3}} \mathcal{A}\left[\varphi_{m}^{2}\right](\mathbf{k})\left[\int_{\eta_{m}}^{\eta_{0}} d \eta \frac{g^{\prime}(\eta)}{g_{m}} j_{\ell}\left(k\left(\eta_{0}-\eta\right)\right)\right] Y_{\ell m}^{*}(\hat{\mathbf{k}}),
$$

\footnotetext{
4 The analyses of the CMB bispectrum performed so far, as for example in Refs. [36, 37], adopt just the linear radiation transfer function, unless the bispectrum originated by specific secondary effects, such as Rees-Sciama or Sunyaev-Zel'dovich effects, is considered.
} 
where we have used that $\varphi_{m}=g_{m} \varphi_{0}$, the relation $4 \pi \sum_{m=-\ell}^{l} Y_{\ell m}^{*}(\hat{\mathbf{k}}) Y_{\ell m}(\hat{\mathbf{n}})=(2 \ell+1) P_{\ell}(\hat{\mathbf{k}} \cdot \hat{\mathbf{n}})$ and the orthonormality of the spherical harmonics. $\mathcal{A}\left[\varphi_{m}^{2}\right](\mathbf{k})$ is a convolution

$$
\mathcal{A}\left[\varphi_{m}^{2}\right](\mathbf{k})=\frac{1}{(2 \pi)^{3}} \int d^{3} k_{1} d^{3} k_{2} \delta^{(3)}\left(\mathbf{k}_{1}+\mathbf{k}_{2}-\mathbf{k}\right)\left[-\frac{10}{3}\left(a_{\mathrm{nl}}-1\right)\right] \varphi_{m}\left(\mathbf{k}_{1}\right) \varphi_{m}\left(\mathbf{k}_{2}\right) .
$$

Following similar steps one can compute the contribution to $a_{\ell m}^{\mathrm{NL}}$ from the other terms in Eq. (3.12). The extension of our example to most of them is straightforward, while for some terms in the (first - order) ${ }_{\text {late }}^{2}$ contribution (3.13) some care must be taken. The details of the computation can be found in Appendix B, and here we report the final result, namely

$$
\begin{aligned}
a_{\ell m}^{\mathrm{NL}} & =4 \pi(-i)^{\ell} \int \frac{d^{3} k}{(2 \pi)^{3}}\left[K_{0}(\mathbf{k}) \Delta_{\ell}^{0(2)}(k)+K_{1}(\mathbf{k}) \Delta_{\ell}^{1(2)}(k)+K_{2}(\mathbf{k}) \Delta_{\ell}^{2(2)}(k)\right] Y_{\ell m}^{*}(\hat{\mathbf{k}}) \\
& +(4 \pi)^{2} \sum_{L_{1} M_{1}} \sum_{L_{2} M_{2}}(-i)^{L_{1}+L_{2}} \mathcal{G}_{\ell L_{1} L_{2}}^{m M_{1} M_{2}} \int \frac{d^{3} k_{1}}{(2 \pi)^{3}} \frac{d^{3} k_{2}}{(2 \pi)^{3}} \varphi_{m}\left(\mathbf{k}_{1}\right) \varphi_{m}\left(\mathbf{k}_{2}\right) \Delta_{L_{1} L_{2}}\left(k_{1}, k_{2}\right) Y_{L_{1} M_{1}}\left(\hat{\mathbf{k}}_{1}\right) Y_{L_{2} M_{2}}\left(\hat{\mathbf{k}}_{2}\right),
\end{aligned}
$$

where $K_{n}(\mathbf{k})$ are convolutions in Fourier space expressed in terms of some kernels $f_{n}\left(\mathbf{k}_{1}, \mathbf{k}_{2}, \mathbf{k}\right)$ as

$$
K_{n}(\mathbf{k})=\frac{1}{(2 \pi)^{3}} \int d^{3} k_{1} d^{3} k_{2} \delta^{(3)}\left(\mathbf{k}_{1}+\mathbf{k}_{2}-\mathbf{k}\right) f_{n}\left(\mathbf{k}_{1}, \mathbf{k}_{2}, \mathbf{k}\right) \varphi_{m}\left(\mathbf{k}_{1}\right) \varphi_{m}\left(\mathbf{k}_{2}\right)
$$

with

$$
\begin{aligned}
& f_{0}\left(\mathbf{k}_{1}, \mathbf{k}_{2}, \mathbf{k}\right)=-\frac{5}{3}\left(a_{\mathrm{n} 1}-1\right)-1, \quad f_{1}\left(\mathbf{k}_{1}, \mathbf{k}_{2}, \mathbf{k}\right)=1 \\
& f_{2}\left(\mathbf{k}_{1}, \mathbf{k}_{2}, \mathbf{k}\right)=3 \frac{\left(\mathbf{k}_{1} \cdot \mathbf{k}\right)\left(\mathbf{k}_{2} \cdot \mathbf{k}\right)}{k^{4}}-\frac{\mathbf{k}_{1} \cdot \mathbf{k}_{2}}{k^{2}}
\end{aligned}
$$

$\mathbf{k}$ given by $\mathbf{k}=\mathbf{k}_{\mathbf{1}}+\mathbf{k}_{\mathbf{2}}$, and correspondingly

$$
\begin{aligned}
& \Delta_{\ell}^{0(2)}(k)=2 \int_{\eta_{m}}^{\eta_{0}} d \eta \frac{g^{\prime}(\eta)}{g_{m}} j_{\ell}\left(k\left(\eta_{0}-\eta\right)\right), \\
& \Delta_{\ell}^{1(2)}(k)=\int_{\eta_{m}}^{\eta_{0}} d \eta \frac{B_{1}^{\prime}(\eta)}{g_{m}^{2}} j_{\ell}\left(k\left(\eta_{0}-\eta\right)\right), \\
& \Delta_{\ell}^{2(2)}(k)=-\int_{\eta_{m}}^{\eta_{0}} d \eta \bar{B}(\eta) j_{\ell}\left(k\left(\eta_{0}-\eta\right)\right) .
\end{aligned}
$$

In Eq. (3.22) $\mathcal{G}_{\ell L_{1} L_{2}}^{m M_{1} M_{2}}=\int d^{2} \mathbf{n} Y_{\ell m}(\hat{\mathbf{n}}) Y_{L_{1} M_{1}}(\hat{\mathbf{n}}) Y_{L_{2} M_{2}}(\hat{\mathbf{n}})$ is the Gaunt integral and ${ }^{5}$

$$
\begin{aligned}
\Delta_{L_{1} L_{2}}\left(k_{1}, k_{2}\right) & =-4 \int_{\eta_{m}}^{\eta_{0}} d \eta \frac{g^{\prime \prime}(\eta)}{g_{m}} j_{L_{1}}\left(k_{1}\left(\eta_{0}-\eta\right)\right) \int_{\eta_{0}}^{\eta} d \tilde{\eta} \frac{g(\tilde{\eta})}{g_{m}} j_{L_{2}}\left(k_{2}\left(\eta_{0}-\tilde{\eta}\right)\right) \\
& +2 \int_{\eta_{m}}^{\eta_{0}} d \eta \frac{g^{\prime}(\eta)}{g_{m}} j_{L_{1}}\left(k_{1}\left(\eta_{0}-\eta\right)\right)\left[2 \int_{\eta_{m}}^{\eta_{0}} d \eta \frac{g^{\prime}(\eta)}{g_{m}} j_{L_{2}}\left(k_{2}\left(\eta_{0}-\eta\right)\right)+\frac{1}{3} j_{L_{2}}\left(k_{2}\left(\eta_{0}-\eta_{*}\right)\right)\right] .
\end{aligned}
$$

Eq. (3.22) is clearly the generalization of the linear relation (3.17) with the functions $\Delta_{l}^{n(2)}(k)$ and $\Delta_{L_{1} L_{2}}\left(k_{1}, k_{2}\right)$ playing the role of coefficients of the second-order radiation transfer function relating quadratic curvature perturbations to the observed temperature anisotropies. ${ }^{6}$ For the second-order ISW effect the transfer function depends on the

\footnotetext{
${ }^{5}$ In fact for the last term of Eq. (3.29), $j_{L_{2}}\left(k_{2}\left(\eta_{0}-\eta_{*}\right)\right) / 3$, corresponding to the last term of Eq. (3.13), we are assuming full matter domination at last scattering $\eta_{*}$, so that we used $\varphi_{*} \approx \varphi_{m}$. However in the final formulae we display in Sec. V we account also for the small differrence between $\varphi_{*}$ and $\varphi_{m}$.

${ }^{6}$ Notice that it is possible to rewrite Eq. (3.22) with a more general formula as
}

$$
a_{\ell m}^{\mathrm{NL}}=(4 \pi)^{2} \sum_{L_{1} M_{1}} \sum_{L_{2} M_{2}}(-i)^{L_{1}+L_{2}} \mathcal{G}_{\ell L_{1} L_{2}}^{m M_{1} M_{2}} \int \frac{d^{3} k_{1}}{(2 \pi)^{3}} \frac{d^{3} k_{2}}{(2 \pi)^{3}} \varphi_{m}\left(\mathbf{k}_{1}\right) \varphi_{m}\left(\mathbf{k}_{2}\right) \Delta_{L_{1} L_{2}}^{\ell}\left(\mathbf{k}_{1}, \mathbf{k}_{2}\right) Y_{L_{1} M_{1}}\left(\hat{\mathbf{k}}_{1}\right) Y_{L_{2} M_{2}}\left(\hat{\mathbf{k}}_{2}\right),
$$


growth suppression factor $g(\eta)$. As we anticipated, for the initial non-Gaussianity, parametrized by $a_{\mathrm{nl}}$ and contained in the quadratic term $K_{0}(\mathbf{k})$, the transfer function $\Delta_{\ell}^{0(2)}(k)$ is exactly the same as in linear theory, while the transfer function becomes non trivial for the remaining terms quadratic in the potential perturbations. In particular the part of $a_{\ell m}^{\mathrm{NL}}$ expressed in terms of the function $\Delta_{L_{1} L_{2}}\left(k_{1}, k_{2}\right)$ comes from the last three terms in Eq. (3.13). Notice that for these contributions the dependence of $\Delta T / T$ on $\hat{\mathbf{n}}$ does not enter only through a single angle $\hat{\mathbf{k}} \cdot \hat{\mathbf{n}}$, as it is usual in linear theory.

\section{Calculation of the early Integrated Sachs-Wolfe effect at second order}

At the epoch $\eta_{*}$ of last scattering the universe is still not completely matter dominated, and the residual radiation component makes the potentials decay in time. Such an effect gives a non negligible contribution to the large-scale CMB anisotropies, since it almost mimics a Sachs-Wolfe effect. For example at the linear level (for a vanishing $\Lambda$ ) the early ISW effect gives a correction to the power spectrum normalization of the order of 20\% [26]. A simple estimate from Eq. (3.8) shows that also at second-order the early integrated effects must be taken into account giving a significant correction to the second-order Sachs-Wolfe effect (1.2).

We consider a universe filled by radiation with energy density $\rho_{\gamma}$ and pressureless matter with energy density $\rho_{m}$, supposing that the cosmological constant term is negligible at the epoch of last scattering. In this case the linear growing mode of the gravitational potential is

$$
\varphi=\varphi_{*} \frac{F(\eta)}{F_{*}}
$$

with $[53,54,55,56]$

$$
F(\eta)=1-\frac{H}{a} \int_{a_{i}}^{a} \frac{d a}{H}=\frac{3}{5}+\frac{2}{15 y}-\frac{8}{15 y^{2}}-\frac{16}{15 y^{3}}+\frac{16 \sqrt{1+y}}{15 y^{3}}
$$

where

$$
y \equiv \frac{\rho_{m}}{\rho_{\gamma}} \propto a
$$

$H(a)$ is the Hubble expansion rate, $a_{i}$ is some epoch when radiation is dominating over matter and the $*$ indicates evaluation at the time of last scattering. Let us briefly recall how to compute the linear early Integrated Sachs-Wolfe effect

$$
a_{\ell m}^{\mathrm{L}}=4 \pi(-i)^{l} \int \frac{d^{3} k}{(2 \pi)^{3}}\left[2 \int_{\eta_{*}}^{\eta_{m}} d \eta \varphi^{\prime}(\mathbf{k}, \eta) j_{\ell}\left(k\left(\eta_{0}-\eta\right)\right)\right] Y_{\ell m}^{*}(\hat{\mathbf{k}})
$$

where the time derivative of the gravitational potential is due to the transition from a residual radiation component to full matter domination reached at the epoch $\eta_{m}$. Since most of the contribution to the early ISW effect comes from near recombination at $\eta_{*}$ the integral (3.35) can be computed by evaluating the Bessel function at $\eta_{*}$ leaving [26]

$$
\left.2 \int_{\eta_{*}}^{\eta_{m}} d \eta \varphi^{\prime}(\mathbf{k}, \eta) \approx 2 \varphi(\mathbf{k}, \eta)\right|_{\eta_{*}} ^{\eta_{m}} j_{\ell}\left(k\left(\eta_{0}-\eta_{*}\right)\right)=2 \varphi_{*}(\mathbf{k}) \frac{\Delta F}{F_{*}} j_{\ell}\left(k\left(\eta_{0}-\eta_{*}\right)\right),
$$

where in the last line we used Eq. (3.32) with $\Delta F=\left.F(\eta)\right|_{\eta_{*}} ^{\eta_{m}}$.

Let us now consider the effect at second-order. The early ISW effect reads

$$
{\frac{\Delta T_{2}}{T}}^{\text {early }}=\left.\frac{1}{2} \int_{\eta_{*}}^{\eta_{m}} d \eta(\Phi+\Psi)^{\prime}(\mathbf{x}, \eta)\right|_{\mathbf{x}=-\hat{\mathbf{n}}\left(\eta_{0}-\eta\right)}+(\text { first }- \text { order })_{\text {early }}^{2}
$$

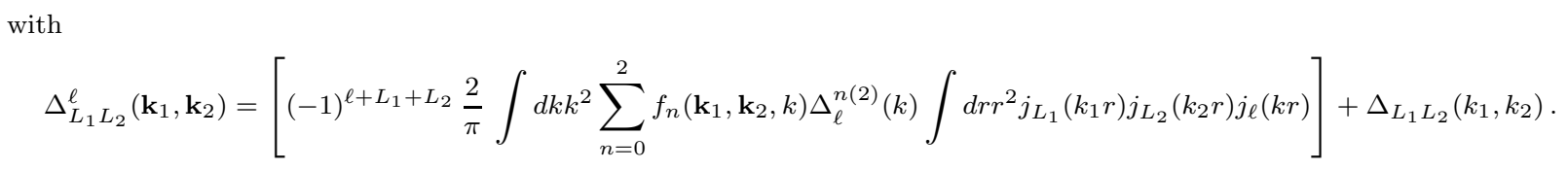


where the (first - order $)_{\text {early }}^{2}$ corrections are obtained from Eq. (3.8) by keeping all those contributions which would vanish in the limit of full matter domination at the epoch of last scattering

$$
\begin{aligned}
(\text { first }- \text { order })_{\text {early }}^{2} & =-4 \int_{\eta_{*}}^{\eta_{m}} d \eta\left[\varphi \varphi^{\prime}+\varphi^{\prime \prime} \int_{\eta_{0}}^{\eta} d \tilde{\eta} \varphi\right]+\frac{1}{2} I_{1}^{2}\left(\eta_{m} ; \eta_{*}\right)+I_{1}\left(\eta_{m} ; \eta_{*}\right) I_{1}\left(\eta_{*}\right)-\frac{1}{3} \varphi_{*} I_{1}\left(\eta_{m} ; \eta_{*}\right) \\
& -4 \varphi_{*}^{\prime} \int_{\eta_{*}}^{\eta_{0}} d \eta\left(\eta_{*}-\eta\right) \varphi
\end{aligned}
$$

where we have splitted $I_{1}\left(\eta_{*}\right)$ defined in Eq. (3.2) as

$$
I_{1}\left(\eta_{*}\right)=-2 \int_{\eta_{*}}^{\eta_{0}} d \eta \varphi^{\prime}=-2 \int_{\eta_{*}}^{\eta_{m}} d \eta \varphi^{\prime}-2 \int_{\eta_{m}}^{\eta_{0}} d \eta \varphi^{\prime} \equiv I_{1}\left(\eta_{m} ; \eta_{*}\right)+I_{1}\left(\eta_{m}\right) .
$$

Notice that, for example, the last term in Eq. (3.38) proportional to $\varphi_{*}^{\prime}$ would give a vanishing contribution in the limit of matter domination when the gravitational potential $\varphi$ is constant in time.

For the moment let us focus on the first term on the R.H.S of Eq. (3.37). The corresponding multipoles are easily computed as in Eq. (3.35)

$$
a_{\ell m}^{\mathrm{NL}}=4 \pi(-i)^{l} \int \frac{d^{3} k}{(2 \pi)^{3}}\left[\frac{1}{2} \int_{\eta_{*}}^{\eta_{m}} d \eta(\Phi+\Psi)^{\prime}(\mathbf{k}, \eta) j_{\ell}\left(k\left(\eta_{0}-\eta\right)\right)\right] Y_{\ell m}^{*}(\hat{\mathbf{k}}),
$$

with the standard approximation

$$
\left.\frac{1}{2} \int_{\eta_{*}}^{\eta_{m}} d \eta(\Phi+\Psi)^{\prime}(\mathbf{k}, \eta) j_{\ell}\left(k\left(\eta_{0}-\eta\right)\right) \simeq \frac{1}{2}(\Phi+\Psi)\right|_{\eta_{*}} ^{\eta_{m}} j_{\ell}\left(k\left(\eta_{0}-\eta_{*}\right)\right) .
$$

Therefore what we need is the expression for the second-order gravitational potentials around the epoch of lastscattering when both radiation and matter are considered. The details of such a computation are reported in Appendix $\mathrm{C}$, where we consider the evolution of the second-order gravitational potentials for a universe filled with radiation and matter.

First of all we find a relation between the gravitational potential $\Phi$ and $\Psi$

$$
\Phi=\Psi+4 \varphi^{2}-\mathcal{Q}
$$

where $\mathcal{Q}$ can be written as

$$
\mathcal{Q}=-\frac{3}{10 F_{*}^{2}} \widetilde{Q} \mathcal{K}
$$

with $\mathcal{K}$ given by Eq. (1.3) and

$$
\widetilde{Q}=-4 \frac{1+y}{4+3 y}\left[\dot{F}^{2}+2 F \dot{F}+\frac{1}{2} \frac{6+5 y}{1+y} F^{2}\right] .
$$

In Eq. (3.44) $y=\rho_{m} / \rho_{\gamma}$ is the ratio of the matter to the radiation component, and a dot stands by a logarithmic derivative with respect to the scale factor, ${ }^{\cdot} \equiv d / d \ln a$. Eq. (3.42) is the analogue of the relation found in in Ref. [25] for a matter-dominated Universe and of Eq. (2.11) (or Eq. (A.11)) for the case of matter plus a cosmological constant.

The evolution of the gravitational potentials is then derived from the equation for $\Psi$

$$
\frac{\sqrt{\rho}}{a}\left[\frac{a}{\sqrt{\rho}} \Psi\right]^{\cdot}=\frac{1}{2} \frac{\dot{\rho}}{\rho} \zeta_{2}+\left[\dot{\varphi}^{2}+\mathcal{Q}-\frac{1}{2} \frac{\dot{\rho}}{\rho} \Delta \zeta_{2}\right],
$$

where $\Delta \zeta_{2}$ is given by Eq. (2.14) evaluated for a system of radiation plus pressureless matter

$$
\frac{1}{2} \frac{\dot{\rho}}{\rho} \Delta \zeta_{2}=\frac{1}{F_{*}^{2}}\left[2 R \dot{F}^{2}-2(2-R) F^{2}-4(1-R) F \dot{F}\right] \varphi_{*}^{2},
$$

and for simplicity we have introduced the function

$$
R(y)=\frac{1+y}{4+3 y}\left(1+\frac{4}{4+3 y}\right) .
$$


Eq. (3.45) has been obtained by using the previous relation (3.42) in the (0-0)-Einstein equation on large scales combined with the expression for the second-order curvature perturbation (2.13) (see Appendix C).

In the case of adiabatic perturbations, for which $\zeta_{2}=$ constant, the integration of Eq. (3.45) yields

$$
\Psi=-F(\eta) \zeta_{2}+\frac{\sqrt{\rho}}{a} \int_{a_{i}}^{a} \frac{d a}{\sqrt{\rho}}\left[\dot{\varphi}^{2}+\mathcal{Q}-\frac{1}{2} \frac{\dot{\rho}}{\rho} \Delta \zeta_{2}\right]+C \frac{\sqrt{\rho}}{a} .
$$

We thus find the usual linear relation plus a source term quadratic in the first-order perturbations. The last term is a decaying mode that can be neglected.

Finally, using Eq. (3.42), we obtain

$$
\begin{aligned}
\Psi+\Phi & =-2 F(\eta) \zeta_{2}+\frac{1}{F_{*}^{2}}\left[4 F^{2}+2 \frac{\sqrt{\rho}}{a} \int_{a_{i}}^{a} \frac{d a}{\sqrt{\rho}}\left((1-2 R) \dot{F}^{2}+2(2-R) F^{2}+4(1-R) F \dot{F}\right)\right] \varphi_{*}^{2} \\
& +\frac{3}{10 F_{*}^{2}}\left[\widetilde{Q}-2 \frac{\sqrt{\rho}}{a} \int_{a_{i}}^{a} \frac{d a}{\sqrt{\rho}} \widetilde{Q}\right] \mathcal{K},
\end{aligned}
$$

which is the expression to be used in Eqs. (3.40) and (3.41) when eavluating the difference $\left.(\Psi+\Phi)\right|_{\eta_{*}} ^{\eta_{m}}$. The conserved quantity $\zeta_{2}$ is determined by the primordial level of non-Gaussianity parametrized as $\zeta_{2}=2 a_{\mathrm{nl}} \zeta_{1}^{2}$, with $\zeta_{1}=-\varphi_{*} / F_{*}$.

To compute the multipoles for the additional terms (3.38) one has to follow the same procedure described in Appendix B. We will use Eq. (3.32) for the epoch when radiation is not negligible (i.e until the time $\eta_{m}$ ) and we will adopt the growth suppression factor $g(\eta)$ as in Eq. (2.3) from full matter domination onwards, with

$$
g_{m} \varphi_{0}=\varphi_{m}=\frac{F_{m}}{F_{*}} \varphi_{*},
$$

where $F_{m}$ denotes the value $F\left(\eta_{m}\right)$. Moreover we will use the evaluation (3.36) for the linear early ISW effect.

Thus we are able to calculate the radiation transfer function for the early ISW effect from Eq. (3.49) and Eq. (3.38). We find

$$
\begin{aligned}
a_{\ell m}^{\mathrm{NL}} & =4 \pi(-i)^{\ell} \int \frac{d^{3} k}{(2 \pi)^{3}}\left[K_{0}(\mathbf{k}) \Delta_{\ell}^{0(2)}(k)+K_{1}(\mathbf{k}) \Delta_{\ell}^{1(2)}(k)+K_{2}(\mathbf{k}) \Delta_{\ell}^{2(2)}(k)\right] Y_{\ell m}^{*}(\hat{\mathbf{k}}) \\
& +(4 \pi)^{2} \sum_{L_{1} M_{1}} \sum_{L_{2} M_{2}}(-i)^{L_{1}+L_{2}} \mathcal{G}_{\ell L_{1} L_{2}}^{m M_{1} M_{2}} \int \frac{d^{3} k_{1}}{(2 \pi)^{3}} \frac{d^{3} k_{2}}{(2 \pi)^{3}} \varphi_{*}\left(\mathbf{k}_{1}\right) \varphi_{*}\left(\mathbf{k}_{2}\right) \Delta_{L_{1} L_{2}}\left(k_{1}, k_{2}\right) Y_{L_{1} M_{1}}\left(\hat{\mathbf{k}}_{1}\right) Y_{L_{2} M_{2}}\left(\hat{\mathbf{k}}_{2}\right)
\end{aligned}
$$

where $\varphi_{*}(\mathbf{k})$ is the gravitational potential at the epoch of last scattering, the convolutions $K_{n}(\mathbf{k})$ are given by

$$
K_{n}(\mathbf{k})=\frac{1}{(2 \pi)^{3}} \int d^{3} k_{1} d^{3} k_{2} \delta^{(3)}\left(\mathbf{k}_{1}+\mathbf{k}_{2}-\mathbf{k}\right) f_{n}\left(\mathbf{k}_{1}, \mathbf{k}_{2}, \mathbf{k}\right) \varphi_{*}\left(\mathbf{k}_{1}\right) \varphi_{*}\left(\mathbf{k}_{2}\right),
$$

with the kernels $f_{n}\left(\mathbf{k}_{1}, \mathbf{k}_{2}, \mathbf{k}\right)$ defined as in Eqs (3.24) and (3.25), and the transfer functions are given by

$$
\begin{aligned}
\Delta_{\ell}^{0(2)}(k) & =\frac{6}{5} \frac{\Delta F}{F_{*}^{2}} j_{\ell}\left(k\left(\eta_{0}-\eta_{*}\right)\right) \\
\Delta_{\ell}^{1(2)}(k) & =\left.\frac{1}{F_{*}^{2}}\left[\frac{\sqrt{\rho}}{a} \int_{a_{i}}^{a} \frac{d a}{\sqrt{\rho}}\left((1-2 R) \dot{F}^{2}+2(2-R) F^{2}+4(1-R) F \dot{F}\right)\right]\right|_{a_{*}} ^{a_{m}} j_{\ell}\left(k\left(\eta_{0}-\eta_{*}\right)\right) \\
& +\frac{4}{5} \frac{\Delta F}{F_{*}^{2}} j_{\ell}\left(k\left(\eta_{0}-\eta_{*}\right)\right) \\
\Delta_{\ell}^{2(2)}(k) & =\left.\frac{1}{F_{*}^{2}}\left[\frac{\widetilde{Q}}{2}-\frac{\sqrt{\rho}}{a} \int_{a_{i}}^{a} \frac{d a}{\sqrt{\rho}} \widetilde{Q}\right]\right|_{a_{*}} ^{a_{m}} j_{\ell}\left(k\left(\eta_{0}-\eta_{*}\right)\right)
\end{aligned}
$$

and

$$
\begin{aligned}
\Delta_{L_{1} L_{2}}\left(k_{1}, k_{2}\right) & =\left(2 \frac{\Delta F^{2}}{F_{*}^{2}}+\frac{2}{3} \frac{\Delta F}{F_{*}}\right) j_{L_{1}}\left(k\left(\eta_{0}-\eta_{*}\right)\right) j_{L_{2}}\left(k\left(\eta_{0}-\eta_{*}\right)\right)+4 \frac{\Delta F}{F_{*}} j_{L_{2}}\left(k\left(\eta_{0}-\eta_{*}\right)\right) \int_{\eta_{m}}^{\eta_{0}} d \eta c \frac{g^{\prime}(\eta)}{g_{m}} j_{L_{1}}\left(k\left(\eta_{0}-\eta\right)\right) \\
& -\frac{F^{\prime}\left(\eta_{*}\right)}{F_{*}} j_{L_{2}}\left(k\left(\eta_{0}-\eta\right)\right)\left[\int_{\eta_{*}}^{\eta_{m}} d \eta\left(\eta_{*}-\eta\right) \frac{F(\eta)}{F_{*}} j_{L_{1}}\left(k\left(\eta_{0}-\eta_{*}\right)\right)+\int_{\eta_{m}}^{\eta_{0}} d \eta\left(\eta_{*}-\eta\right) c \frac{g(\eta)}{g_{m}} j_{L_{1}}\left(k\left(\eta_{0}-\eta\right)\right)\right] \\
& +4 \int_{\eta_{*}}^{\eta_{m}} d \eta \frac{F^{\prime \prime}(\eta)}{F_{*}} j_{L_{2}}\left(k\left(\eta_{0}-\eta\right)\right)\left[\int_{\eta}^{\eta_{m}} d \tilde{\eta} \frac{F(\tilde{\eta})}{F_{*}} j_{L_{1}}\left(k\left(\eta_{0}-\tilde{\eta}\right)\right)+\int_{\eta_{m}}^{\eta_{0}} d \tilde{\eta} \frac{g(\tilde{\eta})}{g_{m}} j_{L_{1}}\left(k\left(\eta_{0}-\tilde{\eta}\right)\right)\right]
\end{aligned}
$$


where $c \equiv F_{m} / F_{*}$ and

$$
\Delta F=F\left(\eta_{m}\right)-F\left(\eta_{*}\right) .
$$

From Eq. (3.53)-(3.56) we see that the second-order early ISW effect can be approximated on large scales as a Sachs-Wolfe effect (whose transfer function is porportional to $j_{\ell}\left(k\left(\eta_{0}-\eta_{*}\right)\right)$ ).

\section{CMB ANISOTROPIES FROM SECOND-ORDER TENSOR PERTURBATIONS}

The second-order tensor contribution to the CMB temperature anisotropies is given by the integrated effect [13]

$$
\frac{\Delta T_{2}}{T}(\mathbf{n})=-\left.\frac{1}{2} \int_{\eta_{*}}^{\eta_{0}} d \eta \chi_{i j}^{(2)^{\prime}}(\mathbf{x}, \eta) n^{i} n^{j}\right|_{\mathbf{x}=-\hat{\mathbf{n}}\left(\eta_{0}-\eta\right)} .
$$

On large (superhorizon) scales the tensor modes remain constant (see, e.g., Ref. [45]), while they start to evolve when they are reentering the cosmological horizon. Therefore the main effect on the CMB anisotropies comes from late times, and therefore we can take $\eta_{*} \approx \eta_{m}$. Notice also that the second-order tensor modes are determined by a contribution produced during inflation and an additional contribution generated by the post-inflationary evolution. Since the level produced during inflation is very small (of the order of the slow-roll parameters) compared to the post-inflationary contribution, as shown for example in Ref. [24], we will neglect it in the following.

For a nonvanishing cosmological constant $\Lambda$ and pressureless matter we show in Appendix A how to obtain the evolution equation for tensor modes

$$
\chi_{2 j}^{i \prime}+2 \mathcal{H} \chi_{2 j}^{i{ }^{\prime}}-\nabla^{2} \chi_{2 j}^{i}=\widetilde{\mathcal{T}}^{i}{ }^{i}
$$

where the source term is given by

$$
\widetilde{\mathcal{T}}_{j}^{i}=4 \nabla^{-2} \partial^{k} \partial_{\ell} R_{k}^{\ell} \delta_{j}^{i}+8 \nabla^{-2}\left(\nabla^{2} R^{i}{ }_{j}-\partial^{i} \partial_{k} R^{k}{ }_{j}-\partial^{k} \partial_{j} R_{k}^{i}\right)+4 \nabla^{-4} \partial^{i} \partial_{j} \partial^{k} \partial_{\ell} R_{k}^{\ell},
$$

and the traceless tensor

$$
\begin{aligned}
R_{k}^{\ell} & \equiv \partial^{\ell} \varphi \partial_{k} \varphi-\frac{1}{3}(\nabla \varphi)^{2} \delta_{k}^{\ell}+4 \pi G a^{2} \bar{\rho}\left(v_{1}^{\ell} v_{1 k}-\frac{1}{3} v_{1}^{2} \delta_{k}^{\ell}\right) \\
& =g^{2}\left(1+\frac{2 E^{2}(z) f^{2}\left(\Omega_{m}\right)}{3 \Omega_{0 m}(1+z)^{3}}\right)\left(\partial^{\ell} \varphi_{0} \partial_{k} \varphi_{0}-\frac{1}{3}\left(\nabla \varphi_{0}\right)^{2} \delta_{k}^{\ell}\right) .
\end{aligned}
$$

It proves convenient to rewrite the source term as

$$
\widetilde{\mathcal{T}}^{i}=s(\eta) \mathcal{T}^{i}{ }_{j}(\mathbf{k})
$$

where

$$
s(\eta)=-8 g^{2}(\eta)\left(1+\frac{2 E^{2}(z) f^{2}\left(\Omega_{m}\right)}{3 \Omega_{0 m}(1+z)^{3}}\right),
$$

and

$$
\nabla^{2} \mathcal{T}_{i j}=\mathcal{S}_{i j} \equiv \nabla^{2} \Theta_{0} \delta_{i j}+\partial_{i} \partial_{j} \Theta_{0}+2\left(\partial_{i} \partial_{j} \varphi_{0} \nabla^{2} \varphi_{0}-\partial_{i} \partial_{k} \varphi_{0} \partial^{k} \partial_{j} \varphi_{0}\right),
$$

with $\Theta_{0}$ defined in Eq. (A.31).

Let us first consider the solution of the second-order tensor modes. We can Fourier transform $\chi_{2 j}^{i}$ and the source term $\widetilde{\mathcal{T}}_{j}^{i}$ and decompose them into the $\sigma=+, \times$ polarization modes,

$$
\begin{aligned}
\chi_{2 j}^{i}(\mathbf{x}, \eta) & =\int \frac{d^{3} \mathbf{k}}{(2 \pi)^{3}} e^{i \mathbf{k} \cdot \mathbf{x}} \chi_{2 j}^{i}(\mathbf{k}, \eta), \\
\chi_{2 j}^{i}(\mathbf{k}, \eta) & =\chi_{+}(\mathbf{k}, \eta) \epsilon_{j}^{+i}(\hat{\mathbf{k}})+\chi_{\times}(\mathbf{k}, \eta) \epsilon_{j}^{\times i}(\hat{\mathbf{k}}),
\end{aligned}
$$


where $\epsilon^{\sigma i}(\hat{\mathbf{k}})$ are the polarization tensors, and similarly for the source term. It is then easy to show that the solution of Eq. (4.2) can be written as

$$
\chi_{2 j}^{i}(\mathbf{k}, \eta)=\mathcal{T}_{j}^{i}(\mathbf{k}) h(k, \eta)
$$

where

$$
h(k, \eta)=\chi_{1}(k, \eta) \int_{\eta_{m}}^{\eta} d \tilde{\eta} \frac{\chi_{2}(k, \tilde{\eta})}{W} s(\tilde{\eta})-\chi_{2}(k, \eta) \int_{\eta_{m}}^{\eta} d \tilde{\eta} \frac{\chi_{2}(k, \tilde{\eta})}{W} s(\tilde{\eta})
$$

and $\chi_{1}$ and $\chi_{2}$ are the solutions for the linear gravity-waves amplitude

$$
\chi^{\prime \prime}+2 \mathcal{H} \chi^{\prime}+k^{2} \chi=0,
$$

$W=\chi_{1}^{\prime} \chi_{2}-\chi_{1} \chi_{2}^{\prime}$ being their corresponding Wronskian. In Eq. (4.10) $\mathcal{T}^{i}{ }_{j}(\mathbf{k})$ represents the amplitude of the secondorder tensor modes fixed by the source term (a quadratic combination of linear scalar modes).

Let us now consider the expression for the multipole coefficients $a_{\ell m}$ for tensor perturbations. We follow some standard References [46, 47, 49] where the reader can find more details on the calculations. From Eq. (3.15) we can define for a given $\mathbf{k}$-mode the coeffiecients

$$
a_{\ell m}(\mathbf{k})=\int d^{2} \mathbf{n} \frac{\Delta T(\mathbf{k}, \hat{\mathbf{n}})}{T} Y_{\ell m}^{*}(\hat{\mathbf{n}}),
$$

where we have expanded in Fourier space the anisotropy $\Delta T(\mathbf{x}, \hat{\mathbf{n}}, \eta) / T=\left(2 \pi^{3}\right)^{-1} \int d^{3} \mathbf{k} e^{i \mathbf{k} \cdot \mathbf{x}} \Delta T(\mathbf{k}, \hat{\mathbf{n}}, \eta) / T$ to be evaluated today and at the origin. Thus from Eq. (4.1) we get for the + polarization state,

$$
a_{\ell m}^{+}(\mathbf{k})=(-1)^{\ell} \int d^{2} \hat{\mathbf{n}} Y_{\ell m}^{*}(\hat{\mathbf{n}})\left[\frac{1}{4} \int_{\eta_{0}}^{\eta_{m}} d \eta \chi_{+}^{\prime}(\mathbf{k}, \eta)\right] \epsilon_{i j}^{+}(\hat{\mathbf{k}}) n^{i} n^{j} \sum_{\ell^{\prime}} i^{\ell^{\prime}}\left(2 \ell^{\prime}+1\right) j_{\ell^{\prime}}\left(k\left(\eta_{0}-\eta\right)\right) P_{\ell^{\prime}}(\hat{\mathbf{n}}),
$$

where we have used the Legendre expansion $e^{i \mathbf{k} \cdot \mathbf{x}}=\sum_{\ell}(2 \ell+1) i^{\ell} j_{\ell}(k x) P_{\ell}(\hat{\mathbf{k}} \cdot \hat{\mathbf{x}})$, and a similar expression holds for the $\times$ polarization state.

It now proves convenient to perform the angular integral for a specific $\mathbf{k}$-mode, by choosing the coordinate system such that $\hat{\mathbf{z}}=\hat{\mathbf{k}}$. Statistical isotropy of observable quantities, like the angular power spectrum or the angle-averaged bispectrum (and higher-order correlation functions) ensures then that we can take the sum over the different modes in $\mathbf{k}$ space at the end. Alternatively we will also display a general formula for a generic $\mathbf{k}$ (and for the coefficients $a_{\ell m}$ ). With such a coordinate system choice the integral in Eq. (4.14) can be evaluated exactly as shown in Refs. [46, 47, 48] to give

$$
\begin{aligned}
a_{\ell m}^{+}(\mathbf{k}) & =(-i)^{\ell} \frac{\sqrt{\pi}}{4} \sqrt{(2 \ell+1)} \sqrt{\frac{(\ell+2) !}{(l-2) !}}\left(\delta_{m 2}+\delta_{m,-2}\right) \int_{\eta_{m}}^{\eta_{0}} d \eta \chi_{+}^{\prime}(\mathbf{k}, \eta) \\
& \times\left[\frac{j_{\ell+2}}{(2 \ell+3)(2 \ell+1)}+2 \frac{j_{\ell}}{(2 \ell+3)(2 \ell-1)}+\frac{j_{\ell-2}}{(2 \ell+1)(2 \ell-1)}\right] \\
& =(-i)^{\ell} \frac{\sqrt{\pi}}{4} \sqrt{(2 \ell+1)} \sqrt{\frac{(\ell+2) !}{(l-2) !}}\left(\delta_{m 2}+\delta_{m,-2}\right) \mathcal{T}_{+}(\mathbf{k}) \int_{\eta_{m}}^{\eta_{0}} d \eta h^{\prime}(k, \eta) \frac{j_{\ell}\left(k\left(\eta_{0}-\eta\right)\right)}{\left[k\left(\eta_{0}-\eta\right)\right]^{2}}
\end{aligned}
$$

where the spherical Bessel functions are evaluted in $k\left(\eta_{0}-\eta\right)$. In the last line we have used Eq. $(4.10), \mathcal{T}_{+}(\mathbf{k})$ being the + component of the amplitude $\mathcal{T}_{j}^{i}(\mathbf{k})$, and we have applied the recursion relation $j_{\ell}(x) / x=\left(j_{\ell-1}(x)+j_{\ell+1}(x)\right) / 2 \ell+1$. For the $\times$ polarization state one has to replace $\left(\delta_{m 2}+\delta_{m,-2}\right)$ with $i\left(\delta_{m,-2}-\delta_{m 2}\right)$.

Following Ref. [48], one can also give a formula holding for a generic k-mode, by using the Wigner-D functions [50] to perform a rotation of the spherical harmonics. One thus finds

$$
\begin{aligned}
a_{\ell m} & =(-i)^{\ell} \frac{\sqrt{\pi}}{4} \sqrt{(2 \ell+1)} \sqrt{\frac{(\ell+2) !}{(l-2) !}} \int d^{3} \mathbf{k} \int_{\eta_{m}}^{\eta_{0}} d \eta \frac{j_{\ell}\left(k\left(\eta_{0}-\eta\right)\right)}{\left[k\left(\eta_{0}-\eta\right)\right]^{2}}\left[\left(\chi_{+}^{\prime}(\mathbf{k}, \eta)-i \chi_{\times}^{\prime}(\mathbf{k}, \eta)\right) D_{m 2}^{l}(\hat{\mathbf{k}})\right. \\
& \left.+\left(\chi_{+}^{\prime}(\mathbf{k}, \eta)+i \chi_{\times}^{\prime}(\mathbf{k}, \eta)\right) D_{m,-2}^{l}(\hat{\mathbf{k}})\right],
\end{aligned}
$$

where $D_{m m^{\prime}}^{l}(\hat{\mathbf{k}})$ are the Wigner-D functions corresponding to a rotation $R(\hat{\mathbf{k}})$ which brings $\hat{\mathbf{k}}$ from $(0,0,1)$ to a generic direction.

The evolution of the second-order tensor perturbations is given by $h(k, \eta)$ having applied the Green method. The solutions for the corresponding homogenous equation (4.12) (which is the one for linear tensor modes) are well known for the limiting cases $\Omega_{m} \rightarrow 1$ and $\Omega_{\Lambda} \rightarrow 1$, and correspondingly analytical expression exist for $\chi_{2 j}^{i}$ (see, e.g., Ref. [28] and [51]). However the full solution requires a numerical evalutation of Eq. (4.12). 


\section{SECOND-ORDER RADIATION TRANSFER FUNCTION FOR LARGE-SCALE CMB ANISOTROPIES: SUMMARY TABLE OF RESULTS AND CONCLUSIONS}

In this section we finalize our computations by providing a summary of the formulae for the full radiation transfer function for the CMB anisotropies on large angular scales due to the second-order Sachs-Wolfe effect in Eq. (1.2), the (early and late) second-order ISW contribution (3.12) and the second-order tensor modes, Eq. (4.1). The section can be regarded as a summary of the main results of this paper.

\section{A. Large-scale CMB anisotropies from second-order scalar perturbations}

The second-order large-scale CMB anisotropies can be written as

$$
\begin{aligned}
a_{\ell m}^{\mathrm{NL}} & =4 \pi(-i)^{\ell} \int \frac{d^{3} k}{(2 \pi)^{3}}\left[K_{0}(\mathbf{k}) \Delta_{\ell}^{0(2)}(k)+K_{1}(\mathbf{k}) \Delta_{\ell}^{1(2)}(k)+K_{2}(\mathbf{k}) \Delta_{\ell}^{2(2)}(k)\right] Y_{\ell m}^{*}(\hat{\mathbf{k}}) \\
& +(4 \pi)^{2} \sum_{L_{1} M_{1}} \sum_{L_{2} M_{2}}(-i)^{L_{1}+L_{2}} \mathcal{G}_{\ell L_{1} L_{2}}^{m M_{1} M_{2}} \int \frac{d^{3} k_{1}}{(2 \pi)^{3}} \frac{d^{3} k_{2}}{(2 \pi)^{3}} \varphi_{*}\left(\mathbf{k}_{1}\right) \varphi_{*}\left(\mathbf{k}_{2}\right) \Delta_{L_{1} L_{2}}\left(k_{1}, k_{2}\right) Y_{L_{1} M_{1}}\left(\hat{\mathbf{k}}_{1}\right) Y_{L_{2} M_{2}}\left(\hat{\mathbf{k}}_{2}\right),
\end{aligned}
$$

where $\varphi_{*}(\mathbf{k})$ is the gravitational potential at the epoch of last scattering, $K_{n}(\mathbf{k})$ are convolutions in Fourier space expressed in terms of some kernels $f\left(\mathbf{k}_{1}, \mathbf{k}_{2}, \mathbf{k}\right)$

$$
K_{n}(\mathbf{k})=\frac{1}{(2 \pi)^{3}} \int d^{3} k_{1} d^{3} k_{2} \delta^{(3)}\left(\mathbf{k}_{1}+\mathbf{k}_{2}-\mathbf{k}\right) f_{n}\left(\mathbf{k}_{1}, \mathbf{k}_{2}, \mathbf{k}\right) \varphi_{*}\left(\mathbf{k}_{1}\right) \varphi_{*}\left(\mathbf{k}_{2}\right),
$$

with

$$
\begin{aligned}
& f_{0}\left(\mathbf{k}_{1}, \mathbf{k}_{2}, \mathbf{k}\right)=-\frac{5}{3}\left(a_{\mathrm{nl}}-1\right)-1 \\
& f_{1}\left(\mathbf{k}_{1}, \mathbf{k}_{2}, \mathbf{k}\right)=1 \\
& f_{2}\left(\mathbf{k}_{1}, \mathbf{k}_{2}, \mathbf{k}\right)=3 \frac{\left(\mathbf{k}_{1} \cdot \mathbf{k}\right)\left(\mathbf{k}_{2} \cdot \mathbf{k}\right)}{k^{4}}-\frac{\mathbf{k}_{1} \cdot \mathbf{k}_{2}}{k^{2}},
\end{aligned}
$$

$\mathbf{k}$ given by $\mathbf{k}=\mathbf{k}_{\mathbf{1}}+\mathbf{k}_{\mathbf{2}}$.

In Eq. (5.3) $a_{\mathrm{nl}}$ is a parameter which specifies the level of primordial non-Gaussianity generated in the early Universe from inflation (or some other alternative mechanisms for the generation of the cosmological perturbations) [8].

The functions $\Delta_{\ell}^{n(2)}(k)$ and $\Delta_{L_{1} L_{2}}\left(k_{1}, k_{2}\right)$ represent the second-order radiation transfer functions, and they are given by the sum of the transfer functions for the Sachs-Wolfe effect (1.2) and the Integrated Sachs-Wolfe effect (3.12).

\section{- Second-order Sachs-Wolfe effect}

Let us first consider the Sachs-Wolfe effect. Notice that the Fourier transform of $3 \mathcal{K} / 10$ corresponds to the convolution $K_{2}(\mathbf{k})$ defined by Eq. (5.2) and Eq. (5.5). Thus it is straightforward to compute the corresponding multipoles for the Sachs-Wolfe effect by using the same steps described in Section III. From Eq. (3.15) we find

$$
\begin{aligned}
\Delta_{\ell}^{0(2)}(k) & =\frac{1}{3} j_{\ell}\left(k\left(\eta_{0}-\eta_{*}\right)\right), \\
\Delta_{\ell}^{1(2)}(k) & =\frac{7}{18} j_{\ell}\left(k\left(\eta_{0}-\eta_{*}\right)\right), \\
\Delta_{\ell}^{2(2)}(k) & =-\frac{1}{3} j_{\ell}\left(k\left(\eta_{0}-\eta_{*}\right)\right), \\
\Delta_{L_{1} L_{2}}\left(k_{1}, k_{2}\right) & \equiv 0 .
\end{aligned}
$$




\section{- Second-order Early ISW effect}

In Section III C we found

$$
\begin{aligned}
\Delta_{\ell}^{0(2)}(k) & =\frac{6}{5} \frac{\Delta F}{F_{*}^{2}} j_{\ell}\left(k\left(\eta_{0}-\eta_{*}\right)\right), \\
\Delta_{\ell}^{1(2)}(k) & =\left.\frac{1}{F_{*}^{2}}\left[\frac{\sqrt{\rho}}{a} \int_{a_{i}}^{a} \frac{d a}{\sqrt{\rho}}\left((1-2 R) \dot{F}^{2}+2(2-R) F^{2}+4(1-R) F \dot{F}\right)\right]\right|_{a_{*}} ^{a_{m}} j_{\ell}\left(k\left(\eta_{0}-\eta_{*}\right)\right) \\
& +\frac{4}{5} \frac{\Delta F}{F_{*}^{2}} j_{\ell}\left(k\left(\eta_{0}-\eta_{*}\right)\right) \\
\Delta_{\ell}^{2(2)}(k) & =\left.\frac{1}{F_{*}^{2}}\left[\frac{\widetilde{Q}}{2}-\frac{\sqrt{\rho}}{a} \int_{a_{i}}^{a} \frac{d a}{\sqrt{\rho}} \widetilde{Q}\right]\right|_{a_{*}} ^{a_{m}} j_{\ell}\left(k\left(\eta_{0}-\eta_{*}\right)\right),
\end{aligned}
$$

and

$$
\begin{aligned}
\Delta_{L_{1} L_{2}}\left(k_{1}, k_{2}\right) & =\left(2 \frac{\Delta F^{2}}{F_{*}^{2}}+\frac{2}{3} \frac{\Delta F}{F_{*}}\right) j_{L_{1}}\left(k\left(\eta_{0}-\eta_{*}\right)\right) j_{L_{2}}\left(k\left(\eta_{0}-\eta_{*}\right)\right) \\
& +4\left[\int_{\eta_{m}}^{\eta_{0}} d \eta c \frac{g^{\prime}(\eta)}{g_{m}} j_{L_{1}}\left(k\left(\eta_{0}-\eta\right)\right)\right] \frac{\Delta F}{F_{*}} j_{L_{2}}\left(k\left(\eta_{0}-\eta_{*}\right)\right) \\
& +4 \int_{\eta_{*}}^{\eta_{m}} d \eta \frac{F^{\prime \prime}(\eta)}{F_{*}} j_{L_{2}}\left(k\left(\eta_{0}-\eta\right)\right)\left[\int_{\eta}^{\eta_{m}} d \tilde{\eta} \frac{F(\tilde{\eta})}{F_{*}} j_{L_{1}}\left(k\left(\eta_{0}-\tilde{\eta}\right)\right)+\int_{\eta_{m}}^{\eta_{0}} d \tilde{\eta} c \frac{g(\tilde{\eta})}{g_{m}} j_{L_{1}}\left(k\left(\eta_{0}-\tilde{\eta}\right)\right)\right] \\
& -\left[\int_{\eta_{*}}^{\eta_{m}} d \eta\left(\eta_{*}-\eta\right) \frac{F(\eta)}{F_{*}} j_{L_{1}}\left(k\left(\eta_{0}-\eta_{*}\right)\right)\right] \frac{F^{\prime}\left(\eta_{*}\right)}{F_{*}} j_{L_{2}}\left(k\left(\eta_{0}-\eta_{*}\right)\right) \\
& -\left[\int_{\eta_{m}}^{\eta_{0}} d \eta\left(\eta_{*}-\eta\right) c \frac{g(\eta)}{g_{m}} j_{L_{1}}\left(k\left(\eta_{0}-\eta\right)\right)\right] \frac{F^{\prime}\left(\eta_{*}\right)}{F_{*}} j_{L_{2}}\left(k\left(\eta_{0}-\eta_{*}\right)\right) .
\end{aligned}
$$

\section{- Second-order Late ISW effect}

From the results of Sections II, III A and III B we have

$$
\begin{aligned}
& \Delta_{\ell}^{0(2)}(k)=2 \int_{\eta_{m}}^{\eta_{0}} d \eta c^{2} \frac{g^{\prime}(\eta)}{g_{m}} j_{\ell}\left(k\left(\eta_{0}-\eta\right)\right), \\
& \Delta_{\ell}^{1(2)}(k)=\int_{\eta_{m}}^{\eta_{0}} d \eta c^{2} \frac{B_{1}^{\prime}(\eta)}{g_{m}^{2}} j_{\ell}\left(k\left(\eta_{0}-\eta\right)\right), \\
& \Delta_{\ell}^{2(2)}(k)=-\int_{\eta_{m}}^{\eta_{0}} d \eta c^{2} \bar{B}(\eta) j_{\ell}\left(k\left(\eta_{0}-\eta\right)\right),
\end{aligned}
$$

and

$$
\begin{aligned}
\Delta_{L_{1} L_{2}}\left(k_{1}, k_{2}\right) & =-4 \int_{\eta_{m}}^{\eta_{0}} d \eta c \frac{g^{\prime \prime}(\eta)}{g_{m}} j_{L_{1}}\left(k_{1}\left(\eta_{0}-\eta\right)\right) \int_{\eta_{0}}^{\eta} d \tilde{\eta} c \frac{g(\tilde{\eta})}{g_{m}} j_{L_{2}}\left(k_{2}\left(\eta_{0}-\tilde{\eta}\right)\right) \\
& +2 \int_{\eta_{m}}^{\eta_{0}} d \eta c \frac{g^{\prime}(\eta)}{g_{m}} j_{L_{1}}\left(k_{1}\left(\eta_{0}-\eta\right)\right)\left[2 \int_{\eta_{m}}^{\eta_{0}} d \eta c \frac{g^{\prime}(\eta)}{g_{m}} j_{L_{2}}\left(k_{2}\left(\eta_{0}-\eta\right)\right)+\frac{1}{3} j_{L_{2}}\left(k_{2}\left(\eta_{0}-\eta_{*}\right)\right] .\right.
\end{aligned}
$$

Let us recall the definition of the different symbols and quantities appearing in the previous formulae.

\section{- Symbols}




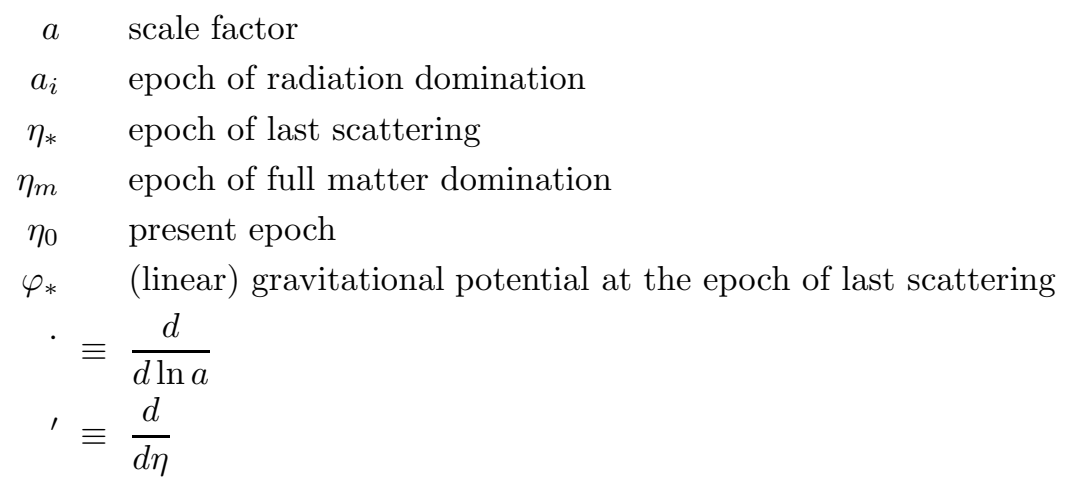

\section{- Functions}

- $F(\eta)$ gives the linear growing-mode solution for the gravitational potential $\varphi$ for adiabatic perturbations of pressurelss matter and radiation fluids $[53,54,55,56]$

$$
F(\eta)=\frac{3}{5}+\frac{2}{15 y}-\frac{8}{15 y^{2}}-\frac{16}{15 y^{3}}+\frac{16 \sqrt{1+y}}{15 y^{3}},
$$

where

$$
y \equiv \frac{\rho_{m}}{\rho_{\gamma}} \propto a,
$$

with

$$
c \equiv F_{m} / F_{*}
$$

and

$$
\Delta F=F\left(\eta_{m}\right)-F\left(\eta_{*}\right) .
$$

- $g(\eta)$ is the growth-suppression factor for a flat Universe filled with a cosmological constant $\Lambda$ and a pressureless fluid whose exact form can be found in Refs. [29, 30,31]. In the $\Lambda=0$ case $g=1$. An excellent approximation for $g$ as a function of redshift $z$ is given in Refs. [29, 30]

$$
g \propto \Omega_{m}\left[\Omega_{m}^{4 / 7}-\Omega_{\Lambda}+\left(1+\Omega_{m} / 2\right)\left(1+\Omega_{\Lambda} / 70\right)\right]^{-1},
$$

with $\Omega_{m}=\Omega_{0 m}(1+z)^{3} / E^{2}(z), \Omega_{\Lambda}=\Omega_{0 \Lambda} / E^{2}(z), E(z) \equiv(1+z) \mathcal{H}(z) / \mathcal{H}_{0}=\left[\Omega_{0 m}(1+z)^{3}+\Omega_{0 \Lambda}\right]^{1 / 2}$ and $\Omega_{0 m}, \Omega_{0 \Lambda}=1-\Omega_{0 m}$, the present-day density parameters of non-relativistic matter and cosmological constant, respectively. We have normalized $g(\eta)$ so that at the present epoch $g(z=0)=1$. The quantity $g_{m}$ corresponds to the value of $g(\eta)$ at the epoch $\eta_{m}$ when full matter domination starts. A good approximation is

$$
g_{m} \approx \frac{2}{5} \Omega_{0 m}^{-1}\left(\Omega_{0 m}^{4 / 7}+\frac{3}{2} \Omega_{0 m}\right) .
$$

- The function $\widetilde{Q}$ enters in the difference between the two second-order gravitational potentials $\Psi$ and $\Phi$, Eq. (3.42), and it is given by

$$
\widetilde{Q}=-4 \frac{1+y}{4+3 y}\left[\dot{F}^{2}+2 F \dot{F}+\frac{1}{2} \frac{6+5 y}{1+y} F^{2}\right]
$$

- The function $R(y)$ has been introduced for simplicty and it is defined as

$$
R(y)=\frac{1+y}{4+3 y}\left(1+\frac{4}{4+3 y}\right)
$$


- The functions $B_{1}(\eta)$ and $B_{2}(\eta)$ enter in the large-scale solution of the gravitational potentials $\Psi$ and $\Phi$, Eqs. (2.22) and (2.23),

$$
\begin{aligned}
& B_{1}(\eta)=\mathcal{H}_{0}^{-2}\left(f_{0}+3 \Omega_{0 m} / 2\right)^{-1} \int_{\eta_{m}}^{\eta} d \tilde{\eta} \mathcal{H}^{2}(\tilde{\eta})(f(\tilde{\eta})-1)^{2} C(\eta, \tilde{\eta}), \\
& B_{2}(\eta)=2 \mathcal{H}_{0}^{-2}\left(f_{0}+3 \Omega_{0 m} / 2\right)^{-1} \int_{\eta_{m}}^{\eta} d \tilde{\eta} \mathcal{H}^{2}(\tilde{\eta})\left[2(f(\tilde{\eta})-1)^{2}-3+3 \Omega_{m}(\tilde{\eta})\right] C(\eta, \tilde{\eta}),
\end{aligned}
$$

where

$$
\begin{aligned}
C(\eta, \tilde{\eta}) & =g^{2}(\tilde{\eta}) a(\tilde{\eta})\left[g(\eta) \mathcal{H}(\tilde{\eta})-g(\tilde{\eta}) \frac{a^{2}(\tilde{\eta})}{a^{2}(\eta)} \mathcal{H}(\eta)\right] \\
f(\eta) & =1+\frac{g^{\prime}(\eta)}{\mathcal{H} g(\eta)} \\
e(\eta) & =\frac{f^{2}(\eta)}{\Omega_{m}(\eta)}
\end{aligned}
$$

- $\bar{B}(\eta)$ is defined by

$$
\bar{B}(\eta)=\left(\frac{B_{2}^{\prime}(\eta)}{g_{m}^{2}}-\frac{4}{3} \frac{g^{\prime}(\eta)}{g_{m}}\right)+\frac{4}{3} \frac{g^{\prime}(\eta) g(\eta)}{g_{m}^{2}}\left(e(\eta)+\frac{3}{2}\right)+\frac{2}{3} \frac{g^{2}(\eta)}{g_{m}^{2}} e^{\prime}(\eta)
$$

\section{B. CMB anisotropies from second-order tensor modes}

In Section IV we found that the contribution of a single k-perturbation mode, with the coordinate system choosen with $\hat{\mathbf{z}}=\hat{\mathbf{k}}$, is given by

$$
\begin{aligned}
& a_{\ell m}^{+}(\mathbf{k})=(-i)^{\ell} \frac{\sqrt{\pi}}{4} \sqrt{(2 \ell+1)} \sqrt{\frac{(\ell+2) !}{(l-2) !}}\left(\delta_{m 2}+\delta_{m,-2}\right) \mathcal{T}_{+}(\mathbf{k}) \int_{\eta_{m}}^{\eta_{0}} d \eta h^{\prime}(k, \eta) \frac{j_{\ell}\left(k\left(\eta_{0}-\eta\right)\right)}{\left[k\left(\eta_{0}-\eta\right)\right]^{2}}, \\
& a_{\ell m}^{\times}(\mathbf{k})=(-i)^{\ell} \frac{\sqrt{\pi}}{4} \sqrt{(2 \ell+1)} \sqrt{\frac{(\ell+2) !}{(l-2) !}} i\left(\delta_{m,-2}-\delta_{m 2}\right) \mathcal{T}_{\times}(\mathbf{k}) \int_{\eta_{m}}^{\eta_{0}} d \eta h^{\prime}(k, \eta) \frac{j_{\ell}\left(k\left(\eta_{0}-\eta\right)\right)}{\left[k\left(\eta_{0}-\eta\right)\right]^{2}},
\end{aligned}
$$

for the + and $\times$ polarization states respectively.

- $\mathcal{T}_{+}(\mathbf{k})$ and $\mathcal{T}_{\times}(\mathbf{k})$ are the + and $\times$ component of the amplitude of the second-order tensor modes, $\mathcal{T}^{i}(\mathbf{k})=$ $\int d^{3} \mathbf{y} e^{-i \mathbf{y} \cdot \mathbf{k}} \mathcal{T}_{j}^{i}(\mathbf{y})$ where

$$
\nabla^{2} \mathcal{T}_{i j}=\nabla^{2} \Theta_{0} \delta_{i j}+\partial_{i} \partial_{j} \Theta_{0}+2\left(\partial_{i} \partial_{j} \varphi_{0} \nabla^{2} \varphi_{0}-\partial_{i} \partial_{k} \varphi_{0} \partial^{k} \partial_{j} \varphi_{0}\right)
$$

with

$$
\nabla^{2} \Theta_{0}=-\frac{1}{2}\left(\left(\nabla^{2} \varphi_{0}\right)^{2}-\partial_{i} \partial_{k} \varphi_{0} \partial^{i} \partial^{k} \varphi_{0}\right)
$$

- $h(k, \eta)$ describes the evolution of the second-order tensor perturbations

$$
h(k, \eta)=\chi_{1}(k, \eta) \int_{\eta_{m}}^{\eta} d \tilde{\eta} \frac{\chi_{2}(k, \tilde{\eta})}{W} s(\tilde{\eta})-\chi_{2}(k, \eta) \int_{\eta_{m}}^{\eta} d \tilde{\eta} \frac{\chi_{2}(k, \tilde{\eta})}{W} s(\tilde{\eta}),
$$

where

$$
s(\eta)=-8 g^{2}(\eta)\left(1+\frac{2 E^{2}(z) f^{2}\left(\Omega_{m}\right)}{3 \Omega_{0 m}(1+z)^{3}}\right),
$$

and $\chi_{1}$ and $\chi_{2}$ are the solutions for the linear tensor modes

$$
\chi^{\prime \prime}+2 \mathcal{H} \chi^{\prime}+k^{2} \chi=0,
$$

$W=\chi_{1}^{\prime} \chi_{2}-\chi_{1} \chi_{2}^{\prime}$ being their corresponding Wronskian. 


\section{Conclusions}

In this paper we have computed the full second-order radiation transfer function for Cosmic Microwave Background anisotropies on large angular scales in a flat universe filled with matter and cosmological constant $\Lambda$. Our calculation includes the second-order generalization of the Sachs-Wolfe effect as well as of (both the early and the late) Integrated Sachs-Wolfe effects, and is valid for a generic set of initial conditions specifying the level of primordial non-Gaussianity. We also accounted for the contribution of second-order tensor modes. Our results deal with large angular scales and represent the first step towards the computation of the full second-order radiation transfer function for CMB anisotropies. Its computation on small angular scales involves several non-linear effects, such as, for example, gravitational lensing, Shapiro time-delay, Rees-Sciama effects, and of course, dealing with the non-linear Boltzmann equation for the photon-matter fluid. Such computation will be the subject of a future publication [19].

\section{Acknowledgments}

We thank Michele Liguori for useful discussions. A. R. is on leave of absence from INFN, Padova (Italy). S. M. acknowledges partial financial support by INAF. N. B. acknowledges partial financial support by INFN.

\section{APPENDIX A: PERTURBATIONS OF A FLAT $\Lambda$ CDM UNIVERSE IN THE POISSON GAUGE.}

\section{Background equations}

Let us consider a flat Friedmann-Robertson-Walker (FRW) universe filled with a pressurelss fluid of energy momentum tensor $T_{\nu}^{\mu}=\rho u^{\mu} u_{\nu}$ plus a cosmological constant $\Lambda$. The Friedmann equations are given by

$$
\begin{aligned}
\mathcal{H}^{2} & =\frac{a^{2}}{3}(8 \pi G \bar{\rho}+\Lambda), \\
\bar{\rho}^{\prime} & =-3 \mathcal{H} \bar{\rho},
\end{aligned}
$$

where $a(\eta)$ is the scale factor, a bar denotes the background quantities and $\mathcal{H}=a^{\prime} / a$, with a prime standing for a derivative with respect to conformal time $\eta$. Another useful relation is

$$
2 \mathcal{H}^{\prime}=-\mathcal{H}^{2}+a^{2} \Lambda
$$

Finally let us recall the definitions of the density parameters $\Omega_{m}=8 \pi G a^{2} \bar{\rho} /\left(3 \mathcal{H}^{2}\right)$ and $\Omega_{\Lambda}=a^{2} \Lambda /(3 \mathcal{H})^{2}$ for the non-relativistic matter component and the cosmological constant respectively, with $\Omega_{m}+\Omega_{\Lambda}=1$.

\section{Linear perturbations}

We briefly report the results for the linear perturbations (for a detailed analysis, see e.g. Ref. [57]). The perturbed metric tensor is defined by Eq. (2.1) and we will adopt the Poisson gauge for which $\omega_{i}$ is a pure vector, i.e. $\partial_{i} \omega^{i}=0$, while $\chi_{i j}$ is a tensor mode (i.e. divergence-free and traceless $\partial^{i} \chi_{i j}=0, \chi_{i}^{i}=0$ ). The matter component has mass density $\rho(\mathbf{x}, \eta)=\bar{\rho}(\eta)(1+\delta)$ and four-velocity $u^{\mu}=\left(\delta_{0}^{\mu}+v^{\mu}\right) / a$, with $u^{\mu} u_{\mu}=-1$. Each perturbation is expanded into a first-order (linear) and second-order part, e.g. for the mass density perturbation $\delta=\delta_{1}(\mathbf{x}, \eta)+\delta_{2}(\mathbf{x}, \eta) / 2$. The traceless part of the $(i-j)$ Einstein's equations in the Poisson gauge imply that $\phi_{1}=\psi_{1} \equiv \varphi$, while its trace gives the evolution equation

$$
\varphi^{\prime \prime}+3 \mathcal{H} \varphi^{\prime}+\left(2 \mathcal{H}^{\prime}+\mathcal{H}^{2}\right) \varphi=0,
$$

which, with Eq. (A.3), brings to Eq. (2.2). Vector modes are not generated from standard mechanisms for cosmological perturbation, such as inflation. Tensor modes obey an evolution equation obtained from the traceless part of the $(i$ j)-component of Einstein equations

$$
\chi_{1 j}^{i}{ }^{\prime \prime}+2 \mathcal{H} \chi_{1 j}^{i}{ }^{\prime}-\nabla^{2} \chi_{1 j}^{i}=0 .
$$


However, for the purposes of the present paper, we can neglect the linear tensor modes in the following.

The energy and momentum constraints provide the density and velocity fluctuations in terms of $\varphi$ (see, for example, Ref. [25] and [15, 28] for the $\Lambda$ case)

$$
\begin{aligned}
\delta_{1} & =\frac{1}{4 \pi G a^{2} \bar{\rho}}\left[\nabla^{2} \varphi-3 \mathcal{H}\left(\varphi^{\prime}+\mathcal{H} \varphi\right)\right], \\
v_{1 i} & =-\frac{1}{4 \pi G a^{2} \bar{\rho}} \partial_{i}\left(\varphi^{\prime}+\mathcal{H} \varphi\right) .
\end{aligned}
$$

\section{Second-order perturbations}

a. Scalar perturbations

Let us first consider the second-order scalar perturbations of the metric in the Poisson gauge $\phi_{2} \equiv \Phi$ and $\psi_{2} \equiv \Psi$. We will now show how to obtain the evolution equation (2.7) for the gravitational potential $\Psi$ and the relation (2.11) which represent our master equations.

The second-order perturbations of the Einstein tensor $G^{\mu}{ }_{\nu}$ can be found for any gauge in Appendix A of Refs. [8, 23]. The perturbations of the energy-momentum tensor up to second order in the Poisson gauge have been computed in Ref. [25] for a general perfect fluid (see Ref. [8] for expressions in any gauge). For the pressureless case one has

$$
\begin{aligned}
& T_{0}^{0}=-\bar{\rho}\left(1+\delta_{1}+\frac{1}{2} \delta_{2}+v_{1}^{2}\right), \\
& T_{0}^{i}=-\bar{\rho}\left[v_{1}^{i}+\frac{1}{2} v_{2}^{i}+\left(\varphi+\delta_{1}\right) v_{1}^{i}\right], \\
& T_{i}^{0}=\bar{\rho}\left[v_{1 i}+\frac{1}{2} v_{2 i}+\frac{1}{2} \omega_{2 i}+\left(-3 \varphi+\delta_{1}\right) v_{1 i}\right], \\
& T_{j}^{i}=\bar{\rho} v_{1}^{i} v_{1 j},
\end{aligned}
$$

where $v_{1}^{2}=v_{1}^{i} v_{1 i}$. Note that the second-order velocity $v_{2 i}$ is the sum of an irrotational component $v_{2 i}^{\|}$, which is the gradient of a scalar, and a rotational vector $v_{2 i}^{\perp}$, which has zero divergence, $\partial^{i} v_{2 i}^{\perp}=0$.

We thus obtain the $(i-j)$-component of Einstein equations

$$
\begin{aligned}
& {\left[\Psi^{\prime \prime}+\mathcal{H}\left(2 \Psi^{\prime}+\Phi^{\prime}\right)+\frac{1}{2} \nabla^{2}(\Phi-\Psi)+\left(2 \mathcal{H}^{\prime}+\mathcal{H}^{2}\right) \Phi-4\left(\mathcal{H}^{\prime}+\mathcal{H}^{2}\right) \varphi^{2}-\varphi^{\prime 2}-8 \mathcal{H} \varphi \varphi^{\prime}-3\left(\partial_{i} \varphi \partial^{i} \varphi\right)-4 \varphi \nabla^{2} \varphi\right] \delta_{j}^{i}} \\
& -\frac{1}{2} \partial^{i} \partial_{j}(\Phi-\Psi)-\frac{1}{2} \mathcal{H}\left(\partial^{i} \omega_{2 j}+\partial_{j} \omega_{2}^{i}\right)-\frac{1}{4}\left(\partial^{i} \omega_{2 j}{ }^{\prime}+\partial_{j} \omega_{2}^{i^{\prime}}\right)+\frac{1}{4}\left[\chi_{2 j}^{i}{ }^{\prime \prime}+2 \mathcal{H} \chi_{2 j}^{i}{ }^{\prime}-\nabla^{2} \chi_{2 j}^{i}\right]+2 \partial^{i} \varphi \partial_{j} \varphi+4 \varphi \partial^{i} \partial_{j} \varphi= \\
& 8 \pi G a^{2} \bar{\rho} v_{1}^{i} v_{1 j} .
\end{aligned}
$$

We now proceed as follows. We take the traceless part of Eq. (A.9), $\delta_{2} G^{i}{ }_{j}-\frac{1}{3} \delta_{2} G_{k}^{k} \delta_{j}^{i}=8 \pi G\left(\delta_{2} T_{j}^{i}-\frac{1}{3} \delta_{2} T_{k}^{k} \delta_{j}^{i}\right)$, namely

$$
\begin{aligned}
& -\left[\frac{1}{6} \nabla^{2}(\Psi-\Phi)+\frac{2}{3}(\nabla \varphi)^{2}+\frac{4}{3} \varphi \nabla^{2} \varphi\right] \delta_{j}^{i}+\frac{1}{2} \partial^{i} \partial_{j}(\Psi-\Phi)+2 \partial^{i} \varphi \partial_{j} \varphi+4 \varphi \partial^{i} \partial_{j} \varphi \\
& -\frac{1}{4}\left(\partial^{i} \omega_{2 j}{ }^{\prime}+\partial_{j} \omega_{2}{ }^{\prime}\right)-\frac{1}{2} \mathcal{H}\left(\partial^{i} \omega_{2 j}+\partial_{j} \omega_{2}^{i}\right)+\frac{1}{4}\left(\chi_{2 j}^{i}{ }^{\prime \prime}+2 \mathcal{H} \chi_{2 j}{ }^{\prime}{ }^{\prime}-\nabla^{2} \chi_{2 j}^{i}\right)=8 \pi G a^{2} \bar{\rho}\left(v_{1}^{i} v_{1 j}-\frac{1}{3} v_{1}^{2} \delta_{j}^{i}\right)
\end{aligned}
$$

and we apply the operator $\partial_{i} \partial^{j}$ in order to get rid of second-order vector and tensor perturbation modes and to solve for the combination $(\Psi-\Phi)$. We find

$$
\Psi-\Phi=-4 \varphi^{2}+\mathcal{Q},
$$

where $\mathcal{Q}$ is defined by

$$
\nabla^{2} \mathcal{Q}=-P+3 N
$$

with

$$
P \equiv P_{i}^{i}
$$


where

$$
P_{j}^{i}=2 \partial^{i} \varphi \partial_{j} \varphi+8 \pi G a^{2} \bar{\rho} v_{1}^{i} v_{1 j}
$$

and the quantity $N$ is given by

$$
\nabla^{2} N \equiv \partial_{i} \partial^{j} P_{j}^{i}
$$

The combination $(\Psi-\Phi)$ will be used in the trace of the $(i-j)$ Einstein equation that we write in the form

$$
\begin{aligned}
\Psi^{\prime \prime}+3 \mathcal{H} \Psi^{\prime}+a^{2} \Lambda \Psi & =\mathcal{H}\left(\Psi^{\prime}-\Phi^{\prime}\right)+a^{2} \Lambda(\Psi-\Phi)+\frac{1}{3} \nabla^{2}(\Psi-\Phi)+4 a^{2} \Lambda \varphi^{2}+\varphi^{\prime 2}+\frac{1}{3}\left(\partial^{i} \varphi \partial_{i} \varphi\right)+8 \mathcal{H} \varphi \varphi^{\prime}+\frac{8}{3} \varphi \nabla^{2} \varphi \\
& +\frac{8 \pi G}{3} \bar{\rho} a^{2} v_{1}^{2}
\end{aligned}
$$

where we have used Eq. (A.3). Inserting Eq. (A.11) into Eq. (A.16) and using Eq. (A.12) and (A.13) we find

$$
\Psi^{\prime \prime}+3 \mathcal{H} \Psi^{\prime}+a^{2} \Lambda \Psi=\mathcal{H} \mathcal{Q}^{\prime}+a^{2} \Lambda \mathcal{Q}+N+\varphi^{\prime 2}-\left(\partial^{i} \varphi \partial_{i} \varphi\right)
$$

The next step is to write explicitly $\mathcal{H} \mathcal{Q}^{\prime}+a^{2} \Lambda \mathcal{Q}$ and $N$ through Eqs. (A.12)-(A.15). Notice that by using Eq. (A.7) one can write $P_{j}^{i}$ as

$$
P_{j}^{i}=\frac{2}{4 \pi G a^{2} \bar{\rho}}\left[\partial^{i} \varphi^{\prime} \partial_{j} \varphi^{\prime}+\mathcal{H}\left(\partial^{i} \varphi \partial_{j} \varphi\right)^{\prime}+\frac{1}{2}\left(5 \mathcal{H}^{2}-a^{2} \Lambda\right) \partial^{i} \varphi \partial_{j} \varphi\right],
$$

where we have also used the background equation (A.1). Thus from Eq. (A.15)

$$
\nabla^{2} \nabla^{2} N=\frac{2}{4 \pi G a^{2} \bar{\rho}} \nabla^{2} \partial_{i} \partial^{j}\left[\partial^{i} \varphi^{\prime} \partial_{j} \varphi^{\prime}+\mathcal{H}\left(\partial^{i} \varphi \partial_{j} \varphi\right)^{\prime}+\frac{1}{2}\left(5 \mathcal{H}^{2}-a^{2} \Lambda\right) \partial^{i} \varphi \partial_{j} \varphi\right] .
$$

For the combination $\mathcal{H} \mathcal{Q}^{\prime}+a^{2} \Lambda \mathcal{Q}$, with the definitions (A.12) and (A.15), we can write

$$
\nabla^{2} \nabla^{2}\left(\mathcal{H} \mathcal{Q}^{\prime}+a^{2} \Lambda \mathcal{Q}\right)=-\nabla^{2}\left(\mathcal{H} P^{\prime}+a^{2} \Lambda P\right)+3 \partial_{i} \partial^{j}\left(\mathcal{H} P^{i}{ }_{j}{ }^{\prime}+a^{2} \Lambda P^{i}{ }_{j}\right) .
$$

Using the background equations (A.1), (A.2) and the evolution equation (2.2) for the linear potential $\varphi$ we get

$$
P_{j}^{i}{ }_{j}^{\prime}=-\frac{2}{4 \pi G a^{2} \bar{\rho}}\left[3 \mathcal{H} \partial^{i} \varphi^{\prime} \partial_{j} \varphi^{\prime}+a^{2} \Lambda \mathcal{H} \partial^{i} \varphi \partial_{j} \varphi+a^{2} \Lambda\left(\partial^{i} \varphi^{\prime} \partial_{j} \varphi+\partial^{i} \varphi \partial_{j} \varphi^{\prime}\right)\right]
$$

and $\mathcal{H} P^{i}{ }_{j}{ }^{\prime}+a^{2} \Lambda P^{i}{ }_{j}=2 a^{2} \Lambda \partial^{i} \varphi \partial_{j} \varphi-4 \partial^{i} \varphi^{\prime} \partial_{j} \varphi^{\prime}$. We then find

$$
\nabla^{2} \nabla^{2}\left(\mathcal{H} \mathcal{Q}^{\prime}+a^{2} \Lambda \mathcal{Q}\right)=4\left[\nabla^{2}\left(\partial_{i} \varphi^{\prime} \partial^{i} \varphi^{\prime}\right)-3 \partial_{i} \partial^{j}\left(\partial^{i} \varphi^{\prime} \partial_{j} \varphi^{\prime}\right)\right]-2 a^{2} \Lambda\left[\nabla^{2}\left(\partial_{i} \varphi \partial^{i} \varphi\right)-3 \partial_{i} \partial^{j}\left(\partial^{i} \varphi \partial_{j} \varphi\right)\right]
$$

We can use the relation $f\left(\Omega_{m}\right) \equiv d \ln D_{+} / d \ln a=1+g^{\prime}(\eta) /(\mathcal{H} g(\eta))$ and Eq. (2.3) to write

$$
\mathcal{H} \mathcal{Q}^{\prime}+a^{2} \Lambda \mathcal{Q}=2 g^{2} \mathcal{H}^{2} \Omega_{m}\left(2 \frac{\left(f\left(\Omega_{m}\right)-1\right)^{2}}{\Omega_{m}}-\frac{3}{\Omega_{m}}+3\right)\left(\nabla^{-2}\left(\partial^{i} \varphi_{0} \partial_{i} \varphi_{0}\right)-3 \nabla^{-4} \partial^{i} \partial_{j}\left(\partial_{i} \varphi_{0} \partial^{j} \varphi_{0}\right)\right)
$$

and

$$
N=\frac{4}{3} g^{2}\left(\frac{f^{2}\left(\Omega_{m}\right)}{\Omega_{m}}+\frac{3}{2}\right) \nabla^{-2} \partial_{i} \partial^{j}\left(\partial^{i} \varphi_{0} \partial_{j} \varphi_{0}\right),
$$

where $\nabla^{-2}$ is the inverse of the Laplacian operator. Therefore, inserting Eqs. (A.23) and (A.24) into Eq. (A.17), we finally obtain Eq. (2.7).

Notice that, in a similar way to $N$, we can write the quantity $P$ defined in Eq. (A.13) as

$$
P=\frac{4}{3} g^{2}\left(\frac{f^{2}\left(\Omega_{m}\right)}{\Omega_{m}}+\frac{3}{2}\right) \partial^{i} \varphi_{0} \partial_{i} \varphi_{0} .
$$

Thus applying the operator $\nabla^{2} \nabla^{2}$ to Eq. (A.11) and combining Eq. (A.25) with Eq. (A.24) we find the relation between $\Psi$ and $\Phi$ of Eq. (2.11). 


\section{b. Vector perturbations}

Let us consider here the second-order calculation which leads to the generation of second-order vector and tensor modes. We will strictly follow the findings of Ref. [28]. For the $\Lambda=0$ case, this problem was originally solved in Ref. [42] starting from the results of second-order calculations in the synchronous gauge [58, 59] and transforming them to the Poisson gauge, by means of second-order gauge transformations [60]. In Ref. [28] the problem is solved directly in the Poisson gauge accounting for a non-vanishing $\Lambda$ term. For a non-zero- $\Lambda$ also Ref. [15] studies second-order vector and tensor modes in the synchronous and Poisson gauges.

We can start by writing the second-order momentum-conservation equation $[61] T_{i ; \mu}^{\mu}=0$, which gives

$$
\left(v_{2 i}^{\prime}+\omega_{2 i}^{\prime}\right)+\mathcal{H}\left(v_{2 i}+\omega_{2 i}\right)=-2 \partial_{i} \Phi-4 \dot{\varphi} v_{1 i}-\partial_{i}\left(v_{1}^{2}+\varphi^{2}\right)
$$

For pure growing-mode initial conditions $\varphi^{\prime} \propto \varphi$ and $v_{1 i} \propto \partial_{i} \varphi$, which makes the RHS of this equation the gradient of a scalar quantity; thus, the vector part only contains a decaying solution $\left(v_{2 i}^{\perp}+\omega_{2 i}\right) \propto a^{-1}$, and we can safely assume $v_{2 i}^{\perp}=-\omega_{2 i}$ (see also Ref. [62] for a discussion on how to obtain the same results using the vorticity vector).

To proceed one needs the second-order perturbations of the Einstein tensor, $\delta_{2} G_{\nu}^{\mu}$ : these can be found for any gauge in Appendix A of Refs. [8, 23].

The second-order 'momentum constraint' $\delta_{2} G_{0}^{i}=8 \pi G \delta_{2} T_{0}^{i}$ gives [28]

$$
\partial^{i}\left(\mathcal{H} \Phi+\Psi^{\prime}\right)-\frac{1}{4} \nabla^{2} \omega^{2 i}+2 \varphi^{\prime} \partial^{i} \varphi+8 \varphi \partial^{i} \varphi^{\prime}=-8 \pi G a^{2} \bar{\rho}\left[\left(\varphi+\delta_{1}\right) v_{1}^{i}+v_{2}^{\| i}\right] .
$$

The pure vector part of this equation can be isolated by first taking its divergence to solve for the combination $\mathcal{H} \Phi+\Psi^{\prime}$ and then replacing it in the original equation. One obtains [28]

$$
-\frac{1}{2} \nabla^{2} \nabla^{2} \omega_{2 i}=16 \pi G a^{2} \bar{\rho} \partial^{j}\left(v_{1 j} \partial_{i} \delta_{1}-v_{1 i} \partial_{j} \delta_{1}\right)
$$

We can further simplify this equation and write:

$$
\nabla^{2} \omega_{2 i}=\frac{4}{3} F(z)\left(\partial_{i} \varphi_{0} \nabla^{2} \varphi_{0}-\partial^{i} \partial^{j} \varphi_{0} \partial_{j} \varphi_{0}+2 \partial_{j} \Theta_{0}\right)
$$

with

$$
F(z)=\frac{2 g^{2}(z) E(z) f\left(\Omega_{m}\right)}{\Omega_{0 m} H_{0}(1+z)^{2}},
$$

where [29, 30] $f\left(\Omega_{m}\right) \equiv d \ln D_{+} / d \ln a \approx \Omega_{m}(z)^{4 / 7}, H_{0}$ is the Hubble constant and

$$
\nabla^{2} \Theta_{0}=-\frac{1}{2}\left(\left(\nabla^{2} \varphi_{0}\right)^{2}-\partial_{i} \partial_{k} \varphi_{0} \partial^{i} \partial^{k} \varphi_{0}\right)
$$

For $\Lambda=0$, the above expression for $\omega_{2 i}$ reduces to Eq. (6.8) of Ref. [42], noting that $F(z)=\eta$ in that case.

\section{c. Tensor perturbations}

For the second-order tensor modes we use the traceless part of the $(i-j)$ Einstein equations, namely Eq. (A.10). To deal with this equation we replace it in the expression for the combination $\Psi-\Phi$, Eq. (2.11), together with the expression for the vector mode $\omega_{2 i}$. After some calculations one arrives at [28]

$$
\nabla^{2} \nabla^{2}\left(\chi_{2 j}^{i}{ }^{\prime \prime}+2 \mathcal{H} \chi_{2 j}^{i}{ }^{\prime}-\nabla^{2} \chi_{2 j}^{i}\right)=4 \nabla^{2} \partial^{k} \partial_{\ell} R_{k}^{\ell} \delta_{j}^{i}+8 \nabla^{2}\left(\nabla^{2} R_{j}^{i}-\partial^{i} \partial_{k} R_{j}^{k}-\partial^{k} \partial_{j} R_{k}^{i}\right)+4 \partial^{i} \partial_{j} \partial^{k} \partial_{\ell} R^{\ell}{ }_{k}
$$

where the traceless tensor

$$
\begin{aligned}
R_{k}^{\ell} & \equiv \partial^{\ell} \varphi \partial_{k} \varphi-\frac{1}{3}(\nabla \varphi)^{2} \delta_{k}^{\ell}+4 \pi G a^{2} \bar{\rho}\left(v_{1}^{\ell} v_{1 k}-\frac{1}{3} v_{1}^{2} \delta_{k}^{\ell}\right) \\
& =g^{2}\left(1+\frac{2 E^{2}(z) f^{2}\left(\Omega_{m}\right)}{3 \Omega_{0 m}(1+z)^{3}}\right)\left(\partial^{\ell} \varphi_{0} \partial_{k} \varphi_{0}-\frac{1}{3}\left(\nabla \varphi_{0}\right)^{2} \delta_{k}^{\ell}\right)
\end{aligned}
$$


Equation (A.32) can be solved by Green's method, as the corresponding homogeneous equation is the one for linear tensor modes, whose analytical solutions are known in the limiting cases $\Omega_{m} \rightarrow 1$ or $\Omega_{\Lambda} \rightarrow 1$. In the $\Lambda \rightarrow 0$ limit one recovers the result of Ref. [42], namely

$$
\chi_{2 i j}(\mathbf{x}, \eta)=\frac{1}{(2 \pi)^{3}} \int d^{3} k e^{i \mathbf{k} \cdot \mathbf{x}} \frac{40}{k^{4}} \mathcal{S}_{i j}(\mathbf{k})\left(\frac{1}{3}-\frac{j_{1}(k \eta)}{k \eta}\right),
$$

where $j_{\ell}$ are spherical Bessel functions of order $\ell$ and $\mathcal{S}_{i j}(\mathbf{k})=\int d^{3} y e^{-i \mathbf{k} \cdot \mathbf{y}} \mathcal{S}_{i j}(\mathbf{y})$, with

$$
\mathcal{S}_{i j}=\nabla^{2} \Theta_{0} \delta_{i j}+\partial_{i} \partial_{j} \Theta_{0}+2\left(\partial_{i} \partial_{j} \varphi_{0} \nabla^{2} \varphi_{0}-\partial_{i} \partial_{k} \varphi_{0} \partial^{k} \partial_{j} \varphi_{0}\right)
$$

\section{APPENDIX B: DERIVATION OF THE MULTIPOLES $a_{\ell m}^{\mathrm{NL}}$}

In this section we will show how to obtain Eq. (3.22). We plug Eq. (3.12) into Eq. (3.15). In Eq. (3.12) the two integrals depend on the time derivative of the second-order gravitational potentials $(\Phi+\Psi)^{\prime}$, while the contribution $(\text { first - order })_{\text {late }}$ comes from additional second-order corrections. For simplicity let us compute these two parts separately in Eq (3.12)

$$
\frac{1}{2}{\frac{\Delta T_{2}}{T}}^{\text {late }}(\hat{\mathbf{n}})=\frac{1}{2}{\frac{\Delta T_{2}}{T}}_{T}\left[\Phi^{\prime}+\Psi^{\prime}\right]+(\text { first }- \text { order })_{\text {late }}^{2},
$$

where

$$
\begin{aligned}
\frac{1}{2} \frac{\Delta T_{2}}{T}\left[\Phi^{\prime}+\Psi^{\prime}\right] & =\int_{\eta_{m}}^{\eta_{0}} d \eta\left[\left(-\frac{10}{3}\left(a_{\mathrm{nl}}-1\right) g_{m} g^{\prime}(\eta)-2 g_{m} g^{\prime}(\eta)+B_{1}^{\prime}(\eta)+4 g(\eta) g^{\prime}(\eta)\right) \varphi_{0}^{2}\right. \\
& \left.+g_{m}^{2} \bar{B}(\eta)\left(\nabla^{-2}\left(\partial^{i} \varphi_{0} \partial_{i} \varphi_{0}\right)-3 \nabla^{-4} \partial_{i} \partial^{j}\left(\partial^{i} \varphi_{0} \partial_{j} \varphi_{0}\right)\right)\right]\left.\right|_{\mathbf{x}=-\hat{\mathbf{n}}\left(\eta_{0}-\eta\right)}
\end{aligned}
$$

here $\bar{B}(\eta)$ is given in Eq. (3.14) and

$$
\begin{aligned}
(\text { first }- \text { order })_{\text {late }}^{2} & =-\left.4 \int_{\eta_{m}}^{\eta_{0}} d \eta\left[g(\eta) g^{\prime}(\eta) \varphi_{0}^{2}(\eta, \mathbf{x})+\left[\int_{\eta_{0}}^{\eta} d \tilde{\eta} \varphi\right] g^{\prime \prime}(\eta) \varphi_{0}(\eta, \mathbf{x})\right]\right|_{\mathbf{x}=-\hat{\mathbf{n}}\left(\eta_{0}-\eta\right)} \\
& +\frac{1}{2} I_{1}^{2}\left(\eta_{m}\right)-\frac{1}{3} \varphi_{*} I_{1}\left(\eta_{m}\right)
\end{aligned}
$$

where we have used Eq. (2.3) into Eq. (3.13). It is important to notice that in Eq. (B.3) $\varphi_{*} \equiv \varphi\left(\eta_{*}, \mathbf{x}=-\hat{\mathbf{n}}\left(\eta_{0}-\eta_{*}\right)\right)$ and the gravitational potential under a given integral must be evaluated along the background geodesics, so that, for example,

$$
\int_{\eta_{0}}^{\eta} d \tilde{\eta} \varphi=\left.\int_{\eta_{0}}^{\eta} d \tilde{\eta} \varphi\right|_{\mathbf{x}=-\hat{\mathbf{n}}\left(\eta_{0}-\tilde{\eta}\right)}=\left.\int_{\eta_{0}}^{\eta} d \tilde{\eta} g^{\prime}(\tilde{\eta}) \varphi_{0}(\tilde{\eta}, \mathbf{x})\right|_{\mathbf{x}=-\hat{\mathbf{n}}\left(\eta_{0}-\tilde{\eta}\right)}
$$

Correspondingly in Eq. (3.15) we will have

$$
a_{l m}^{\mathrm{NL}}=a_{l m}^{\mathrm{NL}}\left[\Phi^{\prime}+\Psi^{\prime}\right]+a_{l m}^{\mathrm{NL}}\left[(\text { first }- \text { order })^{2}\right] .
$$

Let us consider the term in Eq. (B.2). We express it in terms of its Fourier transform

$$
\begin{aligned}
\frac{1}{2} \frac{\Delta T_{2}}{T}\left[\Phi^{\prime}+\Psi^{\prime}\right] & =\int \frac{d^{3} k}{(2 \pi)^{3}} \int_{\eta_{m}}^{\eta_{0}} d \eta\left[\left(-\frac{10}{3}\left(a_{\mathrm{nl}}-1\right) g_{m} g^{\prime}(\eta)-2 g_{m} g^{\prime}(\eta)+B_{1}^{\prime}(\eta)+4 g(\eta) g^{\prime}(\eta)\right)\left[\varphi_{0}^{2}\right](\mathbf{k})\right. \\
& \left.-g_{m}^{2} \bar{B}(\eta) K_{2}(\mathbf{k})\right] e^{-i \mathbf{k} \cdot \hat{\mathbf{n}}\left(\eta_{0}-\eta\right)}
\end{aligned}
$$


where $K_{2}(\mathbf{k})$ is defined in Eq. $(3.23)$ and $\left[\varphi_{0}^{2}\right](\mathbf{k})$ is the convolution giving the Fourier transform of $\varphi_{0}^{2}(\mathbf{x})$. Inserting Eq. (B.6) into Eq. (3.15) and using the Rayleigh expansion

$$
e^{-i \mathbf{k} \cdot \mathbf{x}}=4 \pi \sum_{\ell m}(-i)^{\ell} j_{\ell}(k x) Y_{\ell m}^{*}(\hat{\mathbf{k}}) Y_{\ell m}(\hat{\mathbf{x}})
$$

with the orthonormality of the spherical harmonics we find

$$
\begin{aligned}
a_{l m}^{\mathrm{NL}}\left[\Phi^{\prime}+\Psi^{\prime}\right] & =4 \pi(-i)^{\ell} \int \frac{d^{3} k}{(2 \pi)^{3}} \int_{\eta_{m}}^{\eta_{0}} d \eta j_{\ell}\left(k\left(\eta_{0}-\eta\right)\right)\left[\left(-\frac{10}{3}\left(a_{\mathrm{nl}}-1\right) \frac{g^{\prime}(\eta)}{g_{m}}-2 \frac{g^{\prime}(\eta)}{g_{m}}+\frac{B_{1}^{\prime}(\eta)}{g_{m}^{2}}+4 \frac{g(\eta) g^{\prime}(\eta)}{g_{m}^{2}}\right)\left[\varphi_{0}^{2}\right](\mathbf{k})\right. \\
& \left.-\bar{B}(\eta) K_{2}(\mathbf{k})\right] Y_{l m}^{*}(\hat{\mathbf{k}})
\end{aligned}
$$

where we have used Eq. (2.3).

Let us now consider the term in Eq. (B.3). We Fourier expand the gravitational potential under each integral and we use the Rayleigh expansion (B.7). For the first term in Eq. (B.3) the computation is the same as described above, while the other terms has a different dependence on $\hat{\mathbf{n}}$. For instance, in Eq. (B.3) the integral over $g^{\prime \prime}(\eta)$ becomes

$$
\begin{aligned}
- & \left.4 \int_{\eta_{m}}^{\eta_{0}} d \eta\left[\int_{\eta_{0}}^{\eta} d \tilde{\eta} \varphi\right] g^{\prime \prime}(\eta) \varphi_{0}(\eta, \mathbf{x})\right|_{\mathbf{x}=-\hat{\mathbf{n}}\left(\eta_{0}-\eta\right)}= \\
= & -4 \int \frac{d^{3} k_{1}}{(2 \pi)^{3}} \frac{d^{3} k_{2}}{(2 \pi)^{3}} \int_{\eta_{m}}^{\eta_{0}} d \eta \frac{g^{\prime \prime}(\eta)}{g_{m}} \int_{\eta_{0}}^{\eta} d \tilde{\eta} \frac{g(\tilde{\eta})}{g_{m}} \varphi_{m}\left(\mathbf{k}_{1}\right) \varphi_{m}\left(\mathbf{k}_{2}\right) e^{-i \mathbf{k}_{1} \cdot \hat{\mathbf{n}}\left(\eta_{0}-\eta\right)} e^{-i \mathbf{k}_{2} \cdot \hat{\mathbf{n}}\left(\eta_{0}-\tilde{\eta}\right)} \\
= & -4(4 \pi)^{2} \sum_{L_{1} M_{1}} \sum_{L_{2} M_{2}}(-i)^{L_{1}+L_{2}} Y_{L_{1} M_{1}}^{*}(\hat{\mathbf{n}}) Y_{L_{2} M_{2}}^{*}(\hat{\mathbf{n}}) \int \frac{d^{3} k_{1}}{(2 \pi)^{3}} \frac{d^{3} k_{2}}{(2 \pi)^{3}} \int_{\eta_{m}}^{\eta_{0}} d \eta \frac{g^{\prime \prime}(\eta)}{g_{m}} j_{L_{1}}\left(k_{1}\left(\eta_{0}-\tilde{\eta}\right)\right) \\
& \int_{\eta_{0}}^{\eta} d \tilde{\eta} \frac{g(\tilde{\eta})}{g_{m}} j_{L_{2}}\left(k_{2}\left(\eta_{0}-\tilde{\eta}\right)\right) \varphi_{m}\left(\mathbf{k}_{1}\right) \varphi_{m}\left(\mathbf{k}_{2}\right) Y_{L_{1} M_{1}}\left(\hat{\mathbf{k}}_{1}\right) Y_{L_{2} M_{2}}\left(\hat{\mathbf{k}}_{2}\right),
\end{aligned}
$$

and similar expressions hold for all of the three remaining terms in Eq. (B.3). Eq. (B.9) shows that in general $\Delta T / T$ does not depend on $\hat{\mathbf{n}}$ only through a single angle $\mathbf{k} \cdot \hat{\mathbf{n}}$, as it happens in linear theory.

Thus Eq. (B.3) becomes

$$
\begin{aligned}
& (\text { first - order })_{\text {late }}^{2}=-4 \int \frac{d^{3} k}{(2 \pi)^{3}} \int_{\eta_{*}}^{\eta_{0}} d \eta g(\eta) g^{\prime}(\eta)\left[\varphi_{m}^{2}\right](\mathbf{k}) e^{-i \mathbf{k} \cdot \hat{\mathbf{n}}\left(\eta_{0}-\eta\right)} \\
& +(4 \pi)^{2} \sum_{L_{1} M_{1}} \sum_{L_{2} M_{2}}(-i)^{L_{1}+L_{2}} Y_{L_{1} M_{1}}^{*}(\hat{\mathbf{n}}) Y_{L_{2} M_{2}}^{*}(\hat{\mathbf{n}}) \int \frac{d^{3} k_{1}}{(2 \pi)^{3}} \frac{d^{3} k_{2}}{(2 \pi)^{3}} \varphi_{m}\left(\mathbf{k}_{1}\right) \varphi_{m}\left(\mathbf{k}_{2}\right) \Delta_{L_{1} L_{2}}\left(k_{1}, k_{2}\right) Y_{L_{1} M_{1}}\left(\hat{\mathbf{k}}_{1}\right) Y_{L_{2} M_{2}}\left(\hat{\mathbf{k}}_{2}\right),
\end{aligned}
$$

where $\Delta_{L_{1} L_{2}}\left(k_{1}, k_{2}\right)$ is the function defined in Eq. (3.29). From Eq. (B.10) it is straightforward to compute the corresponding multipoles using Eq. (3.15). We find

$$
\begin{aligned}
a_{l m}^{\mathrm{NL}}\left[(\text { first }- \text { order })^{2}\right] & =-4 \pi(-i)^{\ell} \int \frac{d^{3} k}{(2 \pi)^{3}} \int_{\eta_{*}}^{\eta_{0}} d \eta j_{\ell}\left(k\left(\eta_{0}-\eta\right)\right) \frac{g(\eta) g^{\prime}(\eta)}{g_{m}^{2}}\left[\varphi_{0}^{2}\right](\mathbf{k}) Y_{\ell m}^{*}(\hat{\mathbf{k}}) \\
& +(4 \pi)^{2} \sum_{L_{1} M_{1}} \sum_{L_{2} M_{2}}(-i)^{L_{1}+L_{2}} \mathcal{G}_{\ell L_{1} L_{2}}^{m M_{1} L_{1}} \int \frac{d^{3} k_{1}}{(2 \pi)^{3}} \frac{d^{3} k_{2}}{(2 \pi)^{3}} \varphi_{m}\left(\mathbf{k}_{1}\right) \varphi_{m}\left(\mathbf{k}_{2}\right) \\
& \times \Delta_{L_{1} L_{2}}\left(k_{1}, k_{2}\right) Y_{L_{1} M_{1}}\left(\hat{\mathbf{k}}_{1}\right) Y_{L_{2} M_{2}}\left(\hat{\mathbf{k}}_{2}\right) .
\end{aligned}
$$

The sum of Eq. (B.8) and Eq. (B.11) gives the non-Gaussian part of the multipoles $a_{\ell m}^{\mathrm{NL}}$ for the late ISW effect written in Eq. (3.22). 


\section{APPENDIX C: CALCULATION OF THE EARLY INTEGRATED SACHS-WOLFE EFFECT AT SECOND ORDER}

We consider a flat Universe filled by radiation $\left(\rho_{\gamma}\right)$ and pressureless matter $\left(\rho_{m}\right)$. In this Appendix we derive the equations (3.42) and (3.45).

The relation (3.42) is obtained through the same steps of Section 3 in Appendix A. We take the traceless part of the $(i$ - $j)$-component of Einstein equations at second-order $\delta_{2} G^{i}{ }_{j}-\frac{1}{3} \delta_{2} G_{k}^{k} \delta_{j}^{i}=8 \pi G\left(\delta_{2} T_{j}^{i}-\frac{1}{3} \delta_{2} T_{k}^{k} \delta_{j}^{i}\right)$. The second-order perturbations of the Einstein tensor $G^{\mu}{ }_{\nu}$ can be found for any gauge in Appendix A of Refs. [8, 23]. For the total energy momentum tensor $T_{\mu \nu}=T_{\mu \nu}^{(\gamma)}+T_{\mu \nu}^{(m)}$ one finds

$$
\delta_{2} T^{i}{ }_{j}=\left(\frac{w_{\gamma}}{2} \rho_{\gamma} \delta_{2 \gamma}+\frac{w_{m}}{2} \rho_{m} \delta_{2 m}\right) \delta^{i}{ }_{j}+\rho_{\gamma}\left(1+w_{\gamma}\right) v_{1 \gamma}^{i} v_{j \gamma}^{1}+\rho_{m}\left(1+w_{m}\right) v_{1 m}^{i} v_{j m}^{1}
$$

where $w_{\gamma}=1 / 3$ and $w_{m}=0$ are the equations of state of radiation and matter respectively, $v_{1 \gamma}^{i}, v_{1 m}^{i}$ their linear (scalar) velocities and $\delta_{2 \gamma}=\delta_{2} \rho_{\gamma} / \rho_{\gamma}$. Thus we obtain

$$
\begin{aligned}
& -\left[\frac{1}{6} \nabla^{2}(\Psi-\Phi)+\frac{2}{3}(\nabla \varphi)^{2}+\frac{4}{3} \varphi \nabla^{2} \varphi\right] \delta_{j}^{i}+\frac{1}{2} \partial^{i} \partial_{j}(\Psi-\Phi)+2 \partial^{i} \varphi \partial_{j} \varphi+4 \varphi \partial^{i} \partial_{j} \varphi \\
& -\frac{1}{4}\left(\partial^{i} \omega_{2 j}{ }^{\prime}+\partial_{j} \omega_{2}^{i^{\prime}}\right)-\frac{1}{2} \mathcal{H}\left(\partial^{i} \omega_{2 j}+\partial_{j} \omega_{2}^{i}\right)+\frac{1}{4}\left(\chi_{2 j}{ }^{\prime \prime}+2 \mathcal{H} \chi_{2 j}{ }^{\prime}{ }^{\prime}-\nabla^{2} \chi_{2 j}^{i}\right)= \\
& \frac{3 \mathcal{H}^{2}}{\rho}\left[\rho_{\gamma}\left(1+w_{\gamma}\right) v_{1 \gamma}^{i} v_{j \gamma}^{1}-\frac{1}{3} \rho_{\gamma}\left(1+w_{\gamma}\right) v_{1 \gamma}^{2}+\rho_{m}\left(1+w_{m}\right) v_{1 m}^{i} v_{j m}^{1}-\frac{1}{3} \rho_{m}\left(1+w_{m}\right) v_{1 m}^{2}\right],
\end{aligned}
$$

where $\rho=\rho_{\gamma}+\rho_{m}$ and $v_{1 \gamma}^{2}=v_{1 \gamma}^{i} v_{i \gamma}^{1}$. We now apply the operator $\partial_{i} \partial^{j}$ to get rid of the vector and tensor modes, and to solve for the combination $(\Psi-\Phi)$

$$
\Psi-\Phi=-4 \varphi^{2}+\mathcal{Q}
$$

where $\mathcal{Q}$ is defined by

$$
\nabla^{2} \mathcal{Q}=-P+3 N
$$

with

$$
P \equiv P_{i}^{i}
$$

where

$$
P_{j}^{i}=2 \partial^{i} \varphi \partial_{j} \varphi+3 \mathcal{H}^{2}\left(1+w_{\gamma}\right) \frac{\rho_{\gamma}}{\rho} v_{1 \gamma}^{i} v_{j \gamma}^{1}+3 \mathcal{H}^{2}\left(1+w_{m}\right) \frac{\rho_{m}}{\rho} v_{1 m}^{i} v_{j m}^{1},
$$

and the quantity $N$ is given by

$$
\nabla^{2} N \equiv \partial_{i} \partial^{j} P_{j}^{i}
$$

In order to get the explicit expression for $\mathcal{Q}$, Eq. (3.43), we need to know the linear velocities $v_{j \gamma}^{1}$ and $v_{j m}^{1}$. For adiabatic initial conditions on large scales (deep in the radiation dominated era) one has that initially $v_{j \gamma}^{1}=v_{j m}^{1}$ (see, e.g., Ref. [43]). Moreover it can be shown that on large-scales for adiabatic perturbations the velocities then remain equal (see, e.g. Refs. [53, 64]). Therefore we set $v_{j \gamma}^{1}=v_{j m}^{1} \equiv v_{j}^{1}$ and from the momentum constraint (0- $i$ ) Einstein equation we have the expression of the velocities in term of $\varphi$

$$
\partial_{i}\left(\varphi^{\prime}+\mathcal{H} \varphi\right)=-\frac{3}{2} \mathcal{H}^{2}\left(\frac{4}{3} \frac{\rho_{\gamma}}{\rho} v_{i \gamma}^{1}+\frac{\rho_{m}}{\rho} v_{i m}^{1}\right)=-\frac{3}{2} \mathcal{H}^{2}\left(1+\frac{1}{3} \frac{\rho_{\gamma}}{\rho}\right) v_{i}^{1},
$$

where we have used that $w_{\gamma}=1 / 3$ abd $w_{m}=0$.

Using Eq. (C.8) in Eq. (C.6) brings

$$
P_{j}^{i}=\frac{1}{2 \pi G a^{2} \rho}\left(1+\frac{1}{3} \frac{\rho_{\gamma}}{\rho}\right)^{-1}\left[\partial^{i} \varphi^{\prime} \partial_{j} \varphi^{\prime}+\mathcal{H}\left(\partial^{i} \varphi \partial_{j} \varphi^{\prime}+\partial^{i} \varphi^{\prime} \partial_{j} \varphi\right)+\frac{\mathcal{H}^{2}}{2}\left(5+\frac{\rho_{\gamma}}{\rho}\right) \partial^{i} \varphi \partial_{j} \varphi\right] .
$$


We now make use of the evolution for the linear growing mode of the gravitational potential for adiabatic perturbations $[55]$

$$
\varphi=-F(\eta) \zeta_{1}
$$

where $\zeta_{1}$ is the curvature perturbation which is a constant on large scales for adiabatic perturbations, $\zeta_{1}=$ const. Equivalently we can use Eq. (3.32), with $\zeta_{1}=-\varphi_{*} / F_{*}$. The function $F(\eta)$ is given in Eq. (5.19).

Using Eq. (C.9) we thus find

$$
\begin{aligned}
\nabla^{2} \nabla^{2} N & =\frac{4}{3}\left(1+\frac{\rho_{\gamma}}{\rho}\right)^{-1} \frac{1}{F_{*}^{2}}\left[\left(\frac{F^{\prime}}{\mathcal{H}}\right)^{2}+2 F \frac{F^{\prime}}{\mathcal{H}}+\frac{1}{2}\left(5+\frac{\rho_{\gamma}}{\rho}\right) F^{2}\right] \nabla^{2} \partial_{i} \partial^{j}\left(\partial^{i} \varphi_{*} \partial_{j} \varphi_{*}\right), \\
P & =\frac{4}{3}\left(1+\frac{\rho_{\gamma}}{\rho}\right)^{-1} \frac{1}{F_{*}^{2}}\left[\left(\frac{F^{\prime}}{\mathcal{H}}\right)^{2}+2 F \frac{F^{\prime}}{\mathcal{H}}+\frac{1}{2}\left(5+\frac{\rho_{\gamma}}{\rho}\right) F^{2}\right] \partial^{i} \varphi_{*} \partial_{i} \varphi_{*},
\end{aligned}
$$

and from Eq. (C.4)

$$
\mathcal{Q}=-\frac{4}{3}\left(1+\frac{\rho_{\gamma}}{\rho}\right)^{-1} \frac{1}{F_{*}^{2}}\left[\left(\frac{F^{\prime}}{\mathcal{H}}\right)^{2}+2 F \frac{F^{\prime}}{\mathcal{H}}+\frac{1}{2}\left(5+\frac{\rho_{\gamma}}{\rho}\right) F^{2}\right]\left[\nabla^{-2}\left(\partial^{i} \varphi_{*} \partial_{i} \varphi_{*}\right)-3 \nabla^{-4} \partial_{i} \partial^{j}\left(\partial^{i} \varphi_{*} \partial_{j} \varphi_{*}\right)\right]
$$

Notice that the second squared bracket is $3 \mathcal{K} / 10$, where $\mathcal{K}$ is given by Eq. (1.3). Finally by introducing the ratio $y=\rho_{m} / \rho_{\gamma}$ and using $d / d \ln a=\mathcal{H}^{-1} d / d \eta$ we can write

$$
\mathcal{Q}=-\frac{3}{10 F_{*}^{2}} \widetilde{Q} \mathcal{K}
$$

where $\widetilde{Q}$ is given by Eq. (3.44).

We now describe in some details how to obtain the equation (3.45) for the gravitational potential $\Psi$. We start from the expression for the curvature perturbation $\zeta_{2}$ in the Poisson gauge, see Eq. (2.13),

$$
\zeta_{2}=-\Psi-\mathcal{H} \frac{\delta_{2} \rho}{\rho^{\prime}}+\Delta \zeta_{2}
$$

and the (0-0) Einstein equation on large scales

$$
3 \mathcal{H}\left(\Psi^{\prime}+\mathcal{H} \Phi\right)=8 \pi G \delta_{2} T_{0}^{0}+12 \mathcal{H}^{2} \varphi^{2}+3 \varphi^{\prime 2}
$$

In Eq. (C.15) the second-order corrections $\Delta \zeta_{2}$ are given by

$$
\Delta \zeta_{2}=2 \mathcal{H} \frac{\delta_{1} \rho^{\prime}}{\rho^{\prime}} \frac{\delta_{1} \rho}{\rho^{\prime}}+2 \frac{\delta_{1} \rho}{\rho^{\prime}}\left(\varphi^{\prime}+2 \mathcal{H} \varphi\right)-\left(\frac{\delta_{1} \rho}{\rho^{\prime}}\right)^{2}\left(\mathcal{H} \frac{\rho^{\prime \prime}}{\rho}-\mathcal{H}^{\prime}-2 \mathcal{H}^{2}\right)
$$

while the second-order perturbations of the Einstein tensor $G^{\mu}{ }_{\nu}$ can be found for any gauge in Appendix A of Refs. [8, 23]. The (0-0) component of the energy momentum tensor reads

$$
\delta_{2} T_{0}^{0}=-\frac{1}{2} \delta_{2} \rho-\rho_{m} v_{1 m}^{2}-\frac{4}{3} \rho_{\gamma} v_{1 \gamma}^{2}
$$

where $\rho$ is the total energy density. We thus express $\delta_{2} \rho$ in terms of the gravitational potential through the (0-0) Einstein equation, and we insert it in Eq. (C.18) to find

$$
\zeta_{2}=-\Psi+2 \frac{\rho}{\rho^{\prime}}\left(\Psi^{\prime}+\mathcal{H} \Phi\right)-8 \frac{\rho}{\rho^{\prime}} \mathcal{H} \varphi^{2}-2 \frac{\rho}{\mathcal{H} \rho^{\prime}} \varphi^{\prime 2}+\Delta \zeta_{2},
$$

where we have dropped the velocities appearing in Eq. (C.17) since they are negligible on large scales. Finally we use the relation (C.3) found previously between the two gravitational potentials to eliminate $\Phi$ in Eq. (C.19)

$$
\zeta_{2}=-\Psi+2 \frac{\rho}{\rho^{\prime}}\left(\Psi^{\prime}+\mathcal{H} \Psi\right)-2 \frac{\rho}{\rho^{\prime}} \mathcal{H} \mathcal{Q}-2 \frac{\rho}{\mathcal{H} \rho^{\prime}} \varphi^{\prime 2}+\Delta \zeta_{2} .
$$


Switching time variable from $d / d \eta$ to ${ }^{\cdot} \equiv d / d \ln a=\mathcal{H}^{-1} d / d \eta$ we notice that

$$
-\frac{1}{2} \frac{\dot{\rho}}{\rho} \Psi+(\dot{\Psi}+\Psi)=\frac{\sqrt{\rho}}{a}\left[\frac{a}{\sqrt{\rho}} \Psi\right],
$$

and thus Eq. (C.20) becomes

$$
\frac{\sqrt{\rho}}{a}\left[\frac{a}{\sqrt{\rho}} \Psi\right]=\frac{1}{2} \frac{\dot{\rho}}{\rho} \zeta_{2}+\left[\dot{\varphi}^{2}+\mathcal{Q}-\frac{1}{2} \frac{\dot{\rho}}{\rho} \Delta \zeta_{2}\right] .
$$

In the rest of this Appendix we compute the expression (3.46) for the quantity $\Delta \zeta_{2}$ defined in Eq. (C.17). This is obtained by evaluating $\Delta \zeta_{2}$ in the case of radiation plus pressureless matter.

First of all from the the energy continuity equations $\rho_{\gamma}^{\prime}=-4 \mathcal{H} \rho_{\gamma}$ and $\rho_{m}^{\prime}=-3 \mathcal{H} \rho_{m}$ one obtains for the total energy density $\rho=\rho_{\gamma}+\rho_{m}$

$$
\frac{\rho^{\prime \prime}}{\rho^{\prime}}=\frac{\mathcal{H}^{\prime}}{\mathcal{H}}-\frac{9 \rho_{m}+16 \rho_{\gamma}}{3 \rho_{m}+4 \rho_{\gamma}}
$$

and hence

$$
\mathcal{H} \frac{\rho^{\prime \prime}}{\rho}-\mathcal{H}^{\prime}-2 \mathcal{H}^{2}=-\mathcal{H}^{2}\left(6-\frac{3 \rho_{m}}{3 \rho_{m}+4 \rho_{\gamma}}\right),
$$

which is the combination appearing in Eq. (C.17).

We can now express $\delta_{1} \rho$ and $\delta_{1} \rho^{\prime}$ in terms of the graviational potential $\varphi$ using the total energy continuity equation and the (0-0) Einstein equation in the large scale limit. The total energy continuity equation on large scales reads

$$
\delta_{1} \rho^{\prime}=-3 \mathcal{H}\left(\delta_{1} \rho+\delta_{1} p\right)-3(\rho+p) \varphi^{\prime}=0
$$

where $p=p_{\gamma}+p_{m}=\rho_{\gamma} / 3$, and $\delta_{1} \rho=\delta_{1} \rho_{\gamma}+\delta_{1} \rho_{m}, \delta_{1} p=\delta_{1} p_{\gamma}+\delta_{1} p_{m}=\delta_{1} \rho_{\gamma} / 3$ are the total energy density and pressure perturbation respectively. We now take advantage of the adiabaticity of the perturbations

$$
\frac{\delta_{1} \rho_{\gamma}}{\rho_{\gamma}}=\frac{4}{3} \frac{\delta_{1} \rho_{m}}{\rho_{m}}
$$

to find from the continuity equation

$$
\frac{\delta_{1} \rho^{\prime}}{\rho^{\prime}}=-3 \mathcal{H}\left(1+\frac{1}{3} \frac{4 \rho_{\gamma}}{4 \rho_{\gamma}+3 \rho_{m}}\right) \frac{\delta_{1} \rho}{\rho^{\prime}}-\frac{\varphi^{\prime}}{\mathcal{H}} .
$$

Plugging Eqs. (C.24) and (C.27) into Eq. (C.17) yields

$$
\Delta \zeta_{2}=-\mathcal{H}^{2}\left(\frac{\delta_{1} \rho}{\rho^{\prime}}\right)^{2}\left(1+\frac{4 \rho_{\gamma}}{4 \rho_{\gamma}+3 \rho_{m}}\right)+4 \mathcal{H} \varphi \frac{\delta_{1} \rho}{\rho^{\prime}} .
$$

Finally we use the first-order (0-0) Einstein equation on large scales

$$
\varphi^{\prime}+\mathcal{H} \varphi=-\frac{1}{2} \mathcal{H} \frac{\delta_{1} \rho}{\rho}
$$

to get

$$
\Delta \zeta_{2}=\left[2 \frac{\rho}{\dot{\rho}}(\dot{\varphi}+\varphi)\right]^{2}\left(1+\frac{1}{3} \frac{4 \rho_{\gamma}}{4 \rho_{\gamma}+3 \rho_{m}}\right)-8 \frac{\rho}{\dot{\rho}} \varphi(\dot{\varphi}+\varphi),
$$

where a dot stands by $d / d \ln a$.

We now use Eq. (3.32), which gives linear growing mode for the gravitational potential, to find

$$
\begin{aligned}
-\frac{1}{2} \frac{\dot{\rho}}{\rho} \Delta \zeta_{2} & =\frac{1}{F_{*}^{2}}\left[-2\left(\frac{1+y}{4+3 y}\right)\left(1+\frac{4}{4+3 y}\right) \dot{F}^{2}+2\left(2-\left(\frac{1+y}{4+3 y}\right)\left(1+\frac{4}{4+3 y}\right)\right) F^{2}\right. \\
& \left.+4\left(1-\left(\frac{1+y}{4+3 y}\right)\left(1+\frac{4}{4+3 y}\right)\right) F \dot{F}\right] \varphi_{*}^{2},
\end{aligned}
$$


where $y=\rho_{m} / \rho_{\gamma}$. With the definition

$$
R(y)=\frac{1+y}{4+3 y}\left(1+\frac{4}{4+3 y}\right)
$$

we get Eq. (3.46).

[1] For a review, see D. H. Lyth and A. Riotto, Phys. Rept. 314, 1 (1999).

[2] G. F. Smoot et al., Astrophys. J. 396, L1 (1992).

[3] C. L. Bennett et al., Astrophys. J. 464, L1 (1996).

[4] K. M. Gorski, A. J. Banday, C. L. Bennett, G. Hinshaw, A. Kogut, G. F. Smoot and E. L. Wright, Astrophys. J. 464 (1996) L11.

[5] C. L. Bennett et al., Astrophys. J. Suppl. 148, 1 (2003).

[6] D. N. Spergel et al. [WMAP Collaboration], Astrophys. J. Suppl. 148, 175 (2003).

[7] See http://planck.esa.int/

[8] N. Bartolo, E. Komatsu, S. Matarrese and A. Riotto, Phys. Rept. 402, 103 (2004).

[9] W. Hu, Phys. Rev. D 64, 083005 (2001); T. Okamoto and W. Hu, Phys. Rev. D 66, 063008 (2002).

[10] G. De Troia et al., Mon. Not. Roy. Astron. Soc. 343, 284 (2003).

[11] J. Lesgourgues, M. Liguori, S. Matarrese and A. Riotto, Phys. Rev. D 71, 103514 (2005).

[12] T. Pyne and S. M. Carroll, Phys. Rev. D 53, 2920 (1996).

[13] S. Mollerach and S. Matarrese, Phys. Rev. D 56, 4494 (1997).

[14] N. Bartolo, S. Matarrese and A. Riotto, Phys. Rev. Lett. 93, 231301 (2004).

[15] K. Tomita, Phys. Rev. D 71, 083504 (2005).

[16] K. Tomita, Phys. Rev. D 72, 043526 (2005).

[17] K. Tomita, Phys. Rev. D 72, 103506 (2005).

[18] W. Hu, D. Scott and J. Silk, Phys. Rev. D 49, 648 (1994).

[19] N. Bartolo, M. Liguori, S. Matarrese and A. Riotto, in preparation.

[20] R. K. Sachs and A. M. Wolfe, Astrophys. J. 147, 73 (1967).

[21] N. Bartolo, S. Matarrese and A. Riotto, JCAP 0401, 003 (2004).

[22] N. Bartolo, S. Matarrese and A. Riotto, JCAP 0508, 010 (2005).

[23] V. Acquaviva, N. Bartolo, S. Matarrese and A. Riotto, Nucl. Phys. B 667, 119 (2003).

[24] J. Maldacena, JHEP 0305, 013 (2003).

[25] N. Bartolo, S. Matarrese and A. Riotto, JHEP 0404, 006 (2004).

[26] W. Hu and N. Sugiyama, Astrophys. J. 444, 489 (1995).

[27] M. J. Rees and D. W. Sciama, Nature 517, 611 (1967).

[28] S. Mollerach, D. Harari and S. Matarrese, Phys. Rev. D 69, 063002 (2004).

[29] O. Lahav, P. B. Lilje, J. R. Primack and M. J. Rees, Mon. Not. Roy. Astron. Soc. 251, 128 (1991).

[30] S. M. Carroll, W. H. Press and E. L. Turner, Ann. Rev. Astron. Astrophys. 30, 499 (1992).

[31] D. J. Eisenstein, arXiv:astro-ph/9709054.

[32] K. Enqvist and M. S. Sloth, Nucl. Phys. B 626, 395 (2002); D. H. Lyth and D. Wands, Phys. Lett. B 524, 5 (2002) S. Mollerach, Phys. Rev. D 42, 313 (1990); T. Moroi and T. Takahashi, Phys. Lett. B 522, 215 (2001) [Erratum-ibid. B 539, 303 (2002)].

[33] G. Dvali, A. Gruzinov and M. Zaldarriaga, Phys. Rev. D 69, 023505 (2004).

[34] K. A. Malik and D. Wands, Class. Quant. Grav. 21, L65 (2004).

[35] N. Bartolo, S. Matarrese and A. Riotto, Phys. Rev. D 69, 043503 (2004).

[36] E. Komatsu and D. N. Spergel, Phys. Rev. D 63, 063002 (2001).

[37] E. Komatsu et al., Astrophys. J. Suppl. 148, 119 (2003).

[38] S. Mollerach, A. Gangui, F. Lucchin and S. Matarrese, Astrophys. J. 453, 1 (1995)

[39] D. Munshi, T. Souradeep and A. A. Starobinsky, Astrophys. J. 454, 552 (1995).

[40] D. N. Spergel and D. M. Goldberg, Phys. Rev. D 59, 103001 (1999).

[41] D. M. Goldberg and D. N. Spergel, Phys. Rev. D 59, 103002 (1999).

[42] S. Matarrese, S. Mollerach and M. Bruni, Phys. Rev. D 58, 043504 (1998).

[43] C. P. Ma and E. Bertschinger, Astrophys. J. 455, 7 (1995).

[44] U. Seljak and M. Zaldarriaga, Astrophys. J. 469, 437 (1996).

[45] D. S. Salopek and J. R. Bond, Phys. Rev. D 42, 3936 (1990).

[46] M. J. White, Phys. Rev. D 46, 4198 (1992).

[47] M. Kamionkowski, A. Kosowsky and A. Stebbins, Phys. Rev. D 55, 7368 (1997).

[48] N. Straumann, arXiv:hep-ph/0505249, based on lectures given at the Physik-Combo, in Halle, Leipzig and Jena, $2004 / 2005$.

[49] A. Kosowsky, Annals Phys. 246, 49 (1996).

[50] D. A. Varshalovich, A. N. Moskalev and V. K. Khersonsky, Quantum Theory Of Angular Momentum: Irreducible Tensors, Spherical Harmonics, Vector Coupling Coefficients, 3nj Symbols, World Scientific, Singapore (1988). 
[51] C. Carbone and S. Matarrese, Phys. Rev. D 71, 043508 (2005).

[52] M. Liguori, F. K. Hansen, E. Komatsu, S. Matarrese and A. Riotto, arXiv:astro-ph/0509098.

[53] H. Kodama and M. Sasaki, Prog. Theor. Phys. Suppl. 78, 1 (1984).

[54] H. Kodama and M. Sasaki, Int. J. Mod. Phys. A 1, 265 (1986).

[55] W. Hu and D. J. Eisenstein, Phys. Rev. D 59, 083509 (1999)

[56] S. Dodelson, Modern Cosmology (Academic Press, USA 2003).

[57] V. F. Mukhanov, H. A. Feldman and R. H. Brandenberger, Phys. Rept. 215, 203 (1992).

[58] K. Tomita, Prog. Theor. Phys. 37, 831 (1967).

[59] S. Matarrese, O. Pantano and D. Saez, Phys. Rev. Lett. 72, 320 (1994).

[60] M. Bruni, S. Matarrese, S. Mollerach and S. Sonego, Class. Quant. Grav. 14, 2585 (1997).

[61] Second-order Christoffel symbols can be found in Appendix A of Ref. [8, 23] (see also Ref. [63])

[62] S. Matarrese, S. Mollerach, A. Notari and A. Riotto, Phys. Rev. D 71, 043502 (2005).

[63] H. Noh and J. Hwang, Phys. Rev. D 69104011 (2004).

[64] K. A. Malik and D. Wands, JCAP 0502, 007 (2005) 